\title{
Unique Tracks Drive the Scatter of the Spatially Resolved Star Formation Main Sequence
}

\author{
Christine Hall ${ }^{1}$, Stéphane Courteau ${ }^{1}$ (D), Thomas Jarrett ${ }^{2}$ (1D), Michelle Cluver ${ }^{3,4}$ (i), \\ Gerhardt Meurer ${ }^{5}$, Claude Carignan ${ }^{2}$, and Fiona Audcent-Ross ${ }^{5}$ \\ ${ }^{1}$ Department of Physics, Engineering Physics and Astronomy, Queen's University, Kingston, ON K7L 3N6, Canada \\ ${ }^{2}$ Department of Physics and Astronomy, University of Cape Town, Cape Town, South Africa \\ ${ }^{3}$ Centre for Astrophysics and Supercomputing, Swinburne University of Technology, Hawthorn, VIC 3122, Australia \\ ${ }^{4}$ Department of Physics and Astronomy, University of the Western Cape, Robert Sobukwe Road, Bellville 7535, South Africa \\ ${ }^{5}$ International Centre for Radio Astronomy Research, The University of Western Australia, 35 Stirling Highway, Crawley, WA 6009, Australia \\ Received 2018 May 31; revised 2018 August 16; accepted 2018 September 2; published 2018 October 4
}

\begin{abstract}
The scatter of the spatially resolved star formation main sequence (SFMS) is investigated in order to reveal signatures about the processes of galaxy formation and evolution. We have assembled a sample of 355 nearby galaxies with spatially resolved $\mathrm{H} \alpha$ and mid-infrared fluxes from the Survey for Ionized Neutral Gas in Galaxies and the Wide-field Infrared Survey Explorer, respectively. We examine the impact of various star formation rate (SFR) and stellar mass transformations on the SFMS. Ranging from $10^{6}$ to $10^{11.5} M_{\odot}$ and derived from color to mass-to-light ratio methods for mid-infrared bands, the stellar masses are internally consistent within their range of applicability and inherent systematic errors; a constant mass-to-light ratio also yields representative stellar masses. The various SFR estimates show intrinsic differences and produce noticeable vertical shifts in the SFMS, depending on the timescales and physics encompassed by the corresponding tracer. SFR estimates appear to break down on physical scales below 500 pc. We also examine the various sources of scatter in the spatially resolved SFMS and find morphology does not play a significant role. We identify three unique tracks across the SFMS by individual galaxies, delineated by a critical stellar mass density of log $\left(\Sigma_{M_{*}}\right) \sim 7.5$. Below this scale, the SFMS shows no clear trend and is likely driven by local, stochastic internal processes. Above this scale, all spatially resolved galaxies have comparable SFMS slopes but exhibit two different behaviors, resulting likely from the rate of mass accretion at the center of the galaxy.
\end{abstract}

Key words: galaxies: fundamental parameters - galaxies: photometry - galaxies: spiral - galaxies: star formation galaxies: stellar content - surveys

\section{Introduction}

Studies of the star formation main sequence (SFMS) of galaxies, the relation between star formation rates (SFRs) and stellar mass $\left(M_{*}\right)$, for galaxies throughout our universe have revealed that galaxy formation is an orderly process, and universal laws must govern their evolution throughout cosmological time and across many different environments. Global SFRs rise with cosmic time (Noeske et al. 2007; Whitaker et al. 2014; Salmon et al. 2015; Kelson et al. 2016; Tomczak et al. 2016), while only exhibiting a moderate increase in scatter (Kurczynski et al. 2016; Mitra et al. 2017). Of interest in the study of galaxy scaling relations, like the SFMS, is the ability to separate and identify the processes that lead to their development. Broadly speaking, morphology and galaxy structure correlate strongly with the SFMS. Two well-known, distinct sequences of SFR emerge as typically gauged by $\mathrm{H} \alpha$ emission equivalent widths $(\mathrm{EW}(\mathrm{H} \alpha))$, separating star-forming (blue cloud) and quiescent (red sequence) galaxies (Noeske et al. 2007; Eales et al. 2017; Pandya et al. 2017; Oemler et al. 2017). Similarly, the degree of light concentration, or Sérsic index, is thought to influence the scatter of the global SFMS (Wuyts et al. 2011; Whitaker et al. 2015; Brennan et al. 2017), though its induced shift across the SFMS is more gradual (continuous) than that characterized by the blue cloud and the red sequence. The global environment in which a galaxy evolves also affects its star formation (SF) activity. Indeed, while the relation between environment, from void galaxies to clusters to centrals to satellites, and SF activity in galaxies is non-trivial, there is general agreement that quenching or passive environments prevail in increasingly dense environment (Peng et al. 2012;
Fossati et al. 2015; Beygu et al. 2016; Coil et al. 2017). The processes of gas supply (infalls) and depletion (outflows and consumption) that enable SF may also be traced through the SFMS's apparent dependence on metallicity (Mannucci et al. 2010; Wuyts et al. 2011; Obreja et al. 2014; Telford et al. 2016), or the H I gas fraction (Saintonge et al. 2016).

On large scales, the dark matter halo in which a galaxy is embedded also affects the SFMS through the stellar mass-halo mass $\left(M_{*}-M_{\text {halo }}\right)$ relation ( $\mathrm{Gu}$ et al. 2016; Garrison-Kimmel et al. 2017). Disentangling which of the stellar or halo mass drives this relation is however challenging (Kimm et al. 2009), especially since processes of a different nature dominate in different stellar mass ranges (Williams et al. 2010; Pérez et al. 2013; Beygu et al. 2016; Magdis et al. 2016), with an increasing degree of scatter at lower $M_{*}$ (Guo et al. 2013; Obreja et al. 2014). It remains unclear if the scatter increase at lower $M_{*}$ arises from enhanced stochasticity in lower mass environments, or simply results from the uncertainty in the transformations that are applied. Other studies have also found a correlation

Most studies of the SFMS and its scatter have relied on integrated, global values of the SFR and $M_{*}$ over each galaxy, despite the fact that SF processes display a well-known local dependence, through the star formation, or Kennicutt-Schmidt (K-S), law. ${ }^{6}$ It follows that the gas fraction and surface density should influence the SFMS (Tacconi et al. 2013; Saintonge

\footnotetext{
6 In its most common form, the K-S relation states that star formation densities scale with gas surface densities by $\Sigma_{\mathrm{SFR}} \propto \Sigma_{\text {gas }}^{N}$, where $N=1-2$ is a constant (Schmidt 1959; Kennicutt 1998b; Genzel et al. 2013).
} 
et al. 2016). Since this relationship arises on small scales, one must therefore examine the spatially resolved SFMS, and determine whether the connection between the star formation law and the SFMS holds in progressively smaller regions. Several studies have recently capitalized on the newly available spatially resolved data, probing a local scale SFMS (see Pérez et al. 2013; Wuyts et al. 2013; Hemmati et al. 2014; Cano-Díaz et al. 2016; González Delgado et al. 2016, 2017; Magdis et al. 2016; Abdurro'uf \& Akiyama 2017; Maragkoudakis et al. 2017; Wang et al. 2017; Ellison et al. 2018). Such preliminary spatially resolved studies have demonstrated trends of comparable amplitude between the global and local SFMS properties, such as their slope, zero-points, and scatter, while highlighting that variations in the SFMS on local scale do exist. The morphological dependence of the global SFMS shows latetype galaxies with the highest SFRs defining an upper boundary in the local SFMS, with progressively earlier systems moving across and below the local sequence (González Delgado et al. 2016, 2017; Abdurro'uf \& Akiyama 2017). Intriguingly, the global morphology seems to dictate local structural trends within the galaxy by establishing the vertical offset from the SFMS fit, while maintaining minimal scatter, as measurements from galaxies of the set morphology run along the same SFMS slope.

One of the more powerful observations afforded by spatially resolved studies may arise from examining the shapes of specific SFR ( $\mathrm{SSFR}=\mathrm{SFR} / M_{*}$ ) radial profiles. These profiles are tell-tales of quenching behavior across the galaxy, presenting strong motivation for both inside-out quenching (Forbes et al. 2014; González Delgado et al. 2016; Tacchella et al. 2016b; Belfiore et al. 2018; Ellison et al. 2018, 2018) and outside-in quenching (Schaefer et al. 2017; Medling et al. 2018), and possibly suggesting a shift between inside-out versus outside-in quenching behavior dictated by total $M_{*}$ (Kimm et al. 2009; Pérez et al. 2013; Liu et al. 2018).

Observational studies of the SFMS can also be contrasted with models and simulations at both global and local scales (Dutton et al. 2010; Somerville et al. 2015; Lagos et al. 2016; Rodríguez-Puebla et al. 2016; Tacchella et al. 2016a, 2016b; Brennan et al. 2017; Pandya et al. 2017; Matthee \& Schaye 2018). Simulations can highlight the quenching trends and physical mechanisms that may drive the SFMS over time (Tacchella et al. 2016b; Pandya et al. 2017; Wang et al. 2017); for instance, Tacchella et al. (2016a) surmises that the scatter of the SFMS reflects the oscillation of a galaxy about the SFMS through different evolutionary phases. These phases may ultimately give rise to the separation between star-forming and quiescent sequences (Pandya et al. 2017). Careful datamodel comparisons can indeed complete our understanding of the SFMS scatter.

This study recognizes that spatially resolved investigations of galaxies are the key to understanding the fundamental underpinnings of the SFMS. We wish to identify basic drivers of the SFMS as determined empirically. We can then expand upon the realm of empirical investigations by exploring universal trends on the smallest possible galactic scales. To this end, we wish to examine a comprehensive suite of radial profiles (mass-to-light ratios, stellar masses, SFRs, specific SFRs, and SFR or stellar mass densities) out to larger radial values than most current studies of the global and spatially resolved (local) SFMS. We can also correlate the scatter of the
SFMS with such parameters, and establish connections with numerical models and theoretical investigations accordingly.

The SFMS is characterized by the linear correlation, $\log (\mathrm{SFR})=a \log \left(M_{*}\right)+b$, and great efforts have been invested to measure the variations and dependence of $a$ and $b$. Speagle et al. (2014) provide a comprehensive review of SFMS studies out to $z \approx 6$ with corresponding SFMS fit results for $a$ and $b$ and the relation scatter over the last decade; slope values $(a)$ ranging from 0.05 to 1 , and zero point values $(b)$ ranging from -9.6 to 0.8 are reported. A turnover is commonly observed above $\log \left(M_{*} / M_{\odot}\right) \approx 10.5$ at low redshift, suggesting that the slope $(a)$ decreases at this critical mass (Lee et al. 2015; Tomczak et al. 2016), though there remains some discussion as to whether this is intrinsic to the SFMS or arises from differences between SF and quiescent galaxies (Pearson et al. 2018). The substantial variation in $a$ and $b$ have largely been attributed to the evolution in SFRs over cosmic time; a definitive understanding of the SFMS scatter is, however, still lacking (even though the path that a galaxy follows in the SFMS is clearly a reflection of its mass accretion history and its ability to turn gas into stars across various environments).

In order to study the scatter of the global and local SFMS, our study takes advantage of our own spatially resolved stellar mass maps and SFRs for an ensemble of more than 350 lowredshift galaxies, allowing us to probe local SFR and $M_{*}$ conditions across the galaxy. The variations of the slopes $(a)$, zero point $(b)$, and scatter $(\sigma)$ of the global and local SFMS should yield a clearer appreciation of the processes driving SF on all relevant scales.

The outcome of such spatially resolved SFMS investigations lies critically in the choice of SFR and $M_{*}$ transformations. SFR estimates are made by exploiting spatially resolved $\mathrm{H} \alpha$ flux profiles from the Survey for Ionized Neutral Gas in Galaxies (SINGG; Meurer et al. 2006) and 12 and $23 \mu \mathrm{m}$ band profiles from the Wide-field Infrared Survey Explorer (WISE; Wright et al. 2010). Stellar masses $\left(M_{*}\right)$, which are largely controlled by the integrated SF, can be recovered from the spatially resolved WISE 3.4 and $4.6 \mu \mathrm{m}$ band profiles.

The data and methods to extract SFRs and $M_{*}$ 's are described in Sections 2 and 3. Special attention is paid to the dependence of our results on the choice and consistency of those methods. The influence of the choice of spatial resolution scale is discussed in Section 4. The global and local SFMS is reported in Section 5, and the scatter is analyzed in Sections 6 and 7. Conclusions are summarized in Section 8.

\section{Data}

Our study takes advantage of large, spatially resolved, complementary data sets for two extensive surveys of galaxies: the Survey for Ionization in Neutral Gas Galaxies (SINGG) and WISE.

The SINGG targets were selected from the H I Parkes All Sky Survey (HIPASS), which maps H I $21 \mathrm{~cm}$ line emission, representing regions that likely fuel SF. Of the 468 HIPASS targets, 289 were selected to also be mapped by SINGG. From these targets, 463 distinct galaxies were identified in $\mathrm{H} \alpha$ by these observations, with many HIPASS targets consisting of multiple galaxies (Meurer et al. 2006). SINGG provides resolved $\mathrm{H} \alpha$ and $R$-band maps of these nearby star-forming galaxies (Meurer et al. 2006). These southern hemisphere galaxies have an average redshift of $z \sim 0.01$, with $\mathrm{H}$ I masses in the range of $7.0<\log _{10}\left(M_{*} / M_{\odot}\right)<11.0$ (Hanish et al. 
2006). The subselection from HIPASS favors the nearest galaxies at any given $\mathrm{H}$ I mass. Capitalizing on this subselection alleviates usual optical biases (e.g., total luminosity, surface brightness, or Hubble type; Meurer et al. 2006). A broad range of star-forming environments is thus enabled. The sample has no galaxy inclination cut either. The $\mathrm{H} \alpha$ emission line flux is representative of ionized gas surrounding newly formed, massive stars, and is used as an appropriate tracer for SFR. In addition to galacto-centric radial $\mathrm{H} \alpha$ flux growth profiles and surface brightness profiles, SINGG provides complementary total H I masses.

The WISE data set provides spatially resolved photometry in the $W 1(3.4 \mu \mathrm{m}), W 2(4.6 \mu \mathrm{m}), W 3(12 \mu \mathrm{m})$, and $W 4(23 \mu \mathrm{m})$ bands for galaxies across the whole sky, and overlaps with 355 SINGG galaxies (Jarrett et al. 2013; Brown et al. 2014). The angular resolution of these bands are 5!'9, 6" 5,7 !' 0 , and 12!. 4, respectively (Jarrett et al. 2012). The $W 1$ and $W 2$ bands are sensitive to evolved stellar populations and hence enable the calculation of reliable stellar masses, while the $W 3$ and $W 4$ bands, which have been corrected to remove stellar emission, sample re-radiated light from dust warmed by hot young stars and act as alternative SFR tracers. WISE provides radial flux growth profiles, surface brightness profiles, concentration indices, as well as effective radii and surface brightness for each band. The effective radius, $R_{\text {eff }}$, is the radius within which half the total light of the galaxy is contained. The effective surface brightness, $\mathrm{SB}_{\mathrm{eff}}$, is then the surface brightness at this radius.

These resolved data offer a unique perspective on the global and spatially resolved (local) SFMS. The $\mathrm{H} \alpha$ and WISE radial profiles of each individual galaxy have been rebinned to a matching scale, in order to directly compare each band. WISE resolution can probe regions as small as 90-200 pc across for our closest targets and up to a few kpc for our most distant systems. One must consider whether these spatial scales are a proper match to the transformations being applied and to our theoretical expectations for the correlation between SFR and stellar mass (given that the latter is the integral of the former). The impact and motivation of binning our profiles on various physically meaningful scales are explored in Section 4. Ultimately, our profiles will be rebinned to a spatial scale of 500 pc. With 355 galaxies, this produces a statistically robust local main sequence with nearly 6000 data points.

A tremendous advantage also afforded by these data sets is their large radial extent, reaching out to almost 10 effective radii in some galaxies, unlike most existing surveys, which often only probe out to $1.5 R_{\text {eff. }}$ Our data allow us to trace SFR and $M_{*}$ conditions in commonly unexplored outskirts of a galaxy, reaching low SFRs and surface brightnesses, and encompassing the full variations of SFR and $M_{*}$ across a galaxy.

\section{A Review of Transformation Methods}

\subsection{Star Formation Rates}

There exists a multitude of transformations to infer SFRs in galaxies. These are typically based on UV spectrum slopes, $\mathrm{H} \alpha$ and far-infrared (IR) fluxes, and more (see Kennicutt 1998b; Calzetti 2013; Davies et al. 2016 for a review). These derivations can generally be traced back to motivation by the star formation (or K-S) law, which describes the SFR dependency on the total gas density and photo-reionization models (Schmidt 1959; Kennicutt 1998a, 1998b), though varying ranges of SFRs can be obtained. This variation stems from the use of different wavebands, stellar population models, initial mass functions (IMFs), dust absorption models, and so on applied to each calibration (e.g., Kennicutt 1983). In principle, one single SFR ought to characterize a particular region over a given timescale; the source of SFR differences is also examined below.

The most common transformation is that of Kennicutt (1998b, hereafter K98), where the $\mathrm{H} \alpha$ emission line flux becomes a tracer of instantaneous star formation. This emission arises from ionized hydrogen surrounding a newly formed star, re-emitting the light produced by the young star. This proxy for SF applies to short timescales of $\lesssim 20 \mathrm{Myr}$, as only massive (O and $\mathrm{B}$ ) stars with those ages can heat the gas to suitable temperatures (K98; Davies et al. 2016). Assuming a Salpeter IMF, the SFR conversion is

$$
\operatorname{SFR}_{\mathrm{K} 98, \mathrm{H} \alpha}\left(M_{\odot} \mathrm{yr}^{-1}\right)=7.9 \times 10^{-42} L_{\mathrm{H} \alpha}\left(\mathrm{erg} \mathrm{s}^{-1}\right) .
$$

The largest limitation of this method is accounting for extinction in the $\mathrm{H} \alpha$ emission, which is specific to the environment of study K98.

Young stars also emit copious amounts of ultraviolet photons, which can be absorbed by dust and re-emitted at mid-infrared (MIR) wavelengths, making that light another reliable tracer of SF (Cluver et al. 2014, hereafter C14). The process of light absorption and re-radiation is slow and MIR fluxes typically trace SF over longer timescales of 100 Myr. Cluver et al. (2017, hereafter C17) have developed two SFR conversions motivated by a Kroupa IMF for the WISE W3 $(12 \mu \mathrm{m})$ and $W 4(23 \mu \mathrm{m})$ bands:

$$
\log \left(\mathrm{SFR}_{\mathrm{C} 17, W 3} / M_{\odot} \mathrm{yr}^{-1}\right)=0.889 \log \left(L_{W 3} / L_{\odot}\right)-7.76,
$$

$$
\log \left(\mathrm{SFR}_{\mathrm{C} 17, W 4} / M_{\odot} \mathrm{yr}^{-1}\right)=0.915 \log \left(L_{W 4} / L_{\odot}\right)-8.01 .
$$

If the light is not fully re-radiated, these formulae may actually underestimate the true SFR. The W4 conversion $(23 \mu \mathrm{m}$; Equation (3)) may be preferable over the $W 3(12 \mu \mathrm{m}$; Equation (2)) conversion, as the latter lies within the regime of emission from excited polycyclic aromatic hydrocarbon (PAH) molecules (Jarrett et al. 2013), and therefore has a more complex mapping to SFR. The W4 transformation, which lies beyond the bulk of PAH emission, is therefore favored here. Rosario et al. (2016) compared the corresponding W4 SFRs to those derived from optical tracers (i.e., $\mathrm{H} \alpha$ ) in star-forming galaxies and found comparable agreement. Caution is offered for survey galaxy selections motivated by the $W 4$ band emission, which may bias the analysis toward warm dust regions, and valuable information from cold dust regions would be lost. Fortunately, our target selection was motivated by SINGG, and therefore HIPASS, which is sensitive to $\mathrm{HI}$ emission and unbiased by warm dust.

Yet another SFR transformation by Calzetti et al. (2007, hereafter C07) capitalizes on both $\mathrm{H} \alpha$ and MIPS $24 \mu \mathrm{m}$ to account for dust obscured and unobscured SF environments, in order to produce robust SFR estimates over short and long timescales. Therefore, this calibration should hold over all scales across a galaxy. The hybrid conversion adopts a Kroupa 


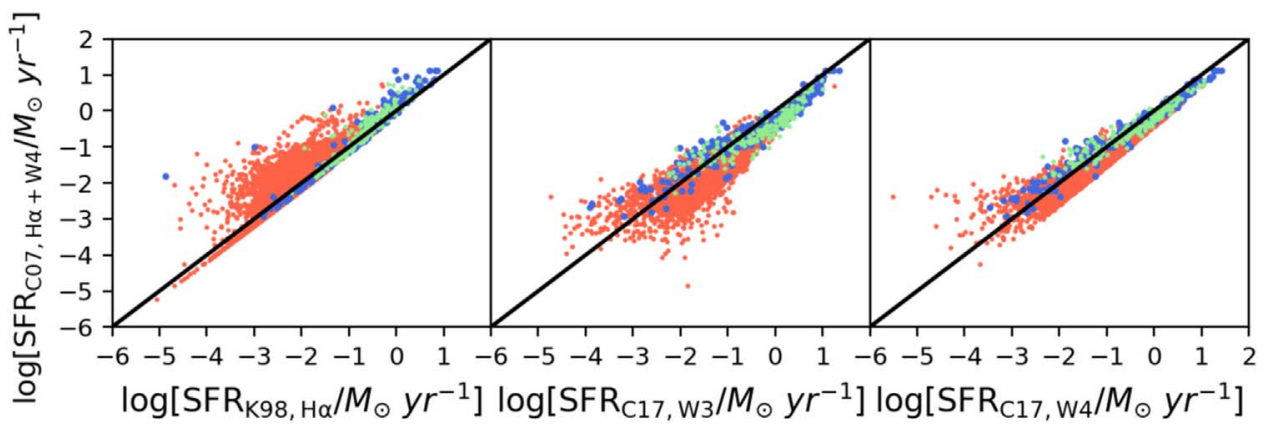

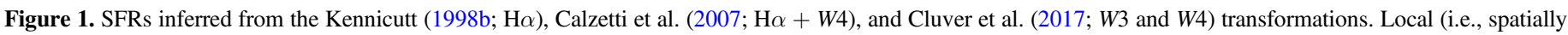

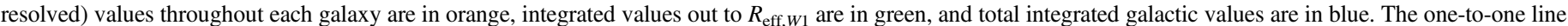
is overlaid in black.

IMF from Starburst99:

$$
\begin{gathered}
\operatorname{SFR}_{\mathrm{C} 07, \mathrm{H} \alpha+24 \mu \mathrm{m}}\left(M_{\odot} \mathrm{yr}^{-1}\right)=5.3 \times 10^{-42}\left[L_{\mathrm{H} \alpha}\left(\mathrm{erg} \mathrm{s}^{-1}\right)\right. \\
\left.+0.031^{*} L_{\nu, 24 \mu \mathrm{m}}\left(\mathrm{erg} \mathrm{s}^{-1} \mathrm{~Hz}^{-1}\right)\right] .
\end{gathered}
$$

This conversion is calibrated to the MIPS $24 \mu \mathrm{m}$ band, which differs slightly from the $W 4$ band; see Figure 2 of Brown et al. (2014). Since these band responses are reasonably consistent, this conversion should be applicable to $W 4$ luminosities, although the differences should be in the sense that SFRs will be slightly underestimated using the W4 band. Fortunately, the overall $W 4$ contribution in this conversion is small, so the impact is arguably negligible.

Calzetti (2013) presents a thorough review and comparison of the many SFR indicators available, examining their motivating physics, unique advantages, and applicable scales. At large scales, single emission line tracers are most reliable under low attenuation conditions. However, as dust contributions increase, as is common in SF galaxies, then a hybrid combination of $\mathrm{H} \alpha$ and $24 \mu \mathrm{m}$ compensates for any attenuated line emission, by accounting for re-radiated dust emission at $24 \mu \mathrm{m}$ (see also, Lee et al. 2013). On increasingly small scales and therefore regions of lower SFR, such indirect tracers become increasingly vulnerable to regions without SF that still emit such light. Across all SFR tracers, the generalization of underlying stellar populations and IMFs that occurs on large scales is likely no longer appropriate. This highlights the complexity and increased vulnerability of tracers at the local, resolved scale. Overall, while each tracer has its own strengths or weaknesses, especially at low SFR regimes, emission line tracers may be the least vulnerable to underlying stellar population assumptions due to the short SF timescales (Calzetti 2013).

Figure 1 compares SFR transformations for both local (orange) and integrated (blue and green) measurements. While global values display some scatter, it is considerably smaller than for local measurements. Innate scatter is expected by the nature of each transformation and the specific SF conditions and timescales of their corresponding tracer represents. However, the increased scatter from global to local values is significant and must be understood. The conversions by $\mathrm{C} 07$ (Equation (4)) and C17 (W4, Equation (3)) produce the most consistent SFRs, while the former transformation has the highest overall agreement among all SFR transformations. This could be due to the fact that $\mathrm{H} \alpha$ and $W 4$ are also in the K98 and $\mathrm{C} 17$, though despite the dominant contribution of $\mathrm{H} \alpha$ in $\mathrm{C} 07$, there is greater consistency with the W4 C17 conversion. The above calibrations, and their impact on the SFMS, are compared in Section 5 in order to isolate the variations in SFR estimates from different tracers. The C07 transformation (Equation (4)) is favored in this analysis since it utilizes both $\mathrm{H} \alpha$, which remains stable across varying stellar populations, and W4 emission, which accounts for dust attenuation effects.

Catalán-Torrecilla et al. (2015) and Davies et al. (2016) also compare different SFR tracers and calibrated transformations. Their calibrations are constructed from CALIFA (Calar Alto Legacy Integral Field sepctroscopy Array) survey and GAMA (Galaxy and Mass Assembly) measurements, respectively. Catalán-Torrecilla et al. (2015) calibrated $\mathrm{H} \alpha$, UV and TIR based transformations, including hybrid combinations of these bands. Using $\mathrm{H} \alpha$ SFRs as the standard to calibrate to, the resulting SFRs from UV and TIR luminosities (or hybrid combinations) are matched to the standard with minimized scatter. The Davies et al. (2016) analysis accounted for $\mathrm{H} \alpha$, $W 3$, and $W 4$ bands as SFR tracers, along with [O II] emission, near and far-ultraviolet, $u$-band, $100 \mu \mathrm{m}$, infrared, and spectral energy distribution fitting. Unique to this study, they examined the consistency of the resulting SFR estimates from each method and their effect on the SFMS. Assuming that SFRs should be the same regardless of the transformation (though this should be cautioned against, as SFRs are dependent on the representative timescale of that tracer), they use SFR calculation discrepancies and SFMS fits to propose revised SFR calibrations for each tracer that ultimately produces SFRs and a SFMS with highest fidelity. The reported hybrid calibration with $\mathrm{H} \alpha$ and $W 4$ by Catalán-Torrecilla et al. (2015) is consistent with the C07 calibration (Equation (4)); the revised calibrations by Davies et al. (2016) are unique. In Section 5, the Davies et al. (2016) calibrations are applied to our data and interpreted in the context of measurements external to the GAMA survey.

\subsection{Stellar Masses}

Stellar masses can be derived through multiple methods; these include color to mass-to-light ratio relations (CMLRs), multiband SED fitting, or straight band flux to stellar mass transformations. Each method takes advantage of specific wavelengths, and this study capitalizes on the availability of the WISE $3.4 \mu \mathrm{m}$ and $4.6 \mu \mathrm{m}$ bands. Jarrett et al. (2013) advocate the advantage of near-infrared light since low mass; evolved stellar populations dominate the emission in that wavelength range and comprise most of a galaxy's stellar mass. With the 


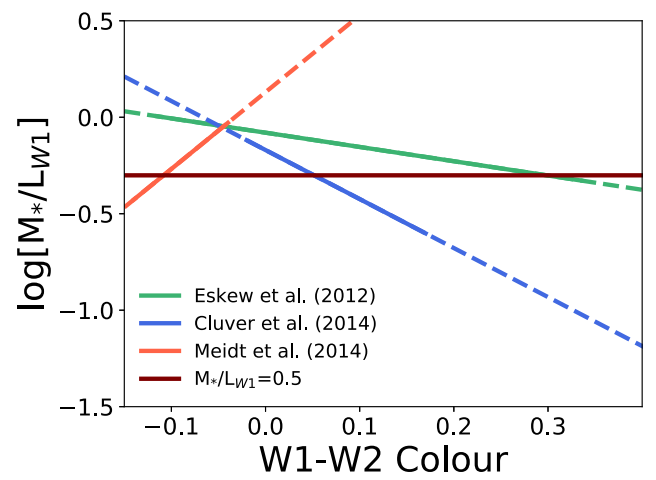

Figure 2. Range of mass-to-light ratios $\left(M_{*} / L_{W 1}\right.$ 's) produced by the stellar transformations by Eskew et al. (2012) in green, Cluver et al. (2014) in blue, and Meidt et al. (2014) in orange. Solid lines represent the applicable color range for each transformation. Dashed lines represent regions where the transformation is no longer physical. The maroon line represents the constant $M_{*} / L_{W 1}=0.5$.

appropriate IMFs and $M_{*} / L$ range, the total stellar mass in a region can be determined from this IR light.

CMLRs have been widely studied, especially for optical bands (Taylor et al. 2011; Roediger \& Courteau 2015; Zhang et al. 2017). Optical $M_{*} / L$ rise with color and dust emission effects are feeble in this wavelength regime. Both Taylor et al. (2011) and Roediger and Courteau (2015) have shown that optical colors result in strongly linear relations since degeneracy from dust, age, or metallicity scatter along the relation, rather than away it. They use SED fitting (though with different algorithms) to derive stellar masses and extract CMLRs. Roediger and Courteau (2015) emphasize, however, that the reliability of $M_{*}$ estimates improves with the number of bands utilized, thus favoring SED fitting.

An increase in dust emission in SF galaxies translates to a reddening of MIR colors, with $W 1-W 2>0$. This reddening is unlike that for optical colors from low-mass stars (which would correspond to increasing $M_{*} / L_{W 1}$ ), whereas red MIR colors lower $M_{*} / L_{W 1}$ as the light emission is largely dominated by dust (Querejeta et al. 2015). One must distinguish whether the detected emission has been corrected for dust effects in order to apply the appropriate calibration to stellar mass. MIR CMLRs are highly sensitive to dust and the presence of dust changes mechanisms driving the relation between the MIR color and $M_{*} / L_{W 1}$. Such trends are demonstrated in Figure 2. If $W 1-W 2>0$, dust is typically present. Although Wright et al. (2010) and C14 show in their Figures 12 and 5, respectively, that the range of $W 1-W 2$ colors corresponding to spiral galaxies is roughly between -0.2 and 0.6 , values exceeding $W 1-W 2=0.3$ represent AGNs or starbursts and should be avoided (Stern et al. 2012, C14).

These considerations are critical in seeking accurate stellar masses from the WISE (MIR) data. Before selecting a stellar mass transformation for this analysis, the methods utilizing the 3.4 and $4.6 \mu \mathrm{m}$ bands, we first compare the methods that apply for the 3.4 and $4.6 \mu \mathrm{m}$ bands. We enforce that each transformation caters to different galactic environments (E12, C14, M14) and note that these CMLR conversions can be applied only for a specific $W 1-W 2$ color range.

The stellar mass conversion by $\mathrm{C} 14$ improves upon the preliminary WISE conversion by Jarrett et al. (2013). The original transformation was derived using a Charbrier-type IMF that takes into account general models of star formation histories, stellar population age and composition, dust content, and AGN activity. Improvements by C14 use in-depth calibrations of GAMA stellar mass estimates by Taylor et al. (2011) for more than 110,000 low redshift (z) galaxies. It represents a robust sampling of galactic environments, similar to our study. The conversion is meant to apply to "normal" galaxies and avoids warmer regions that may contain AGN or starbursting environments where the relation breaks down (beyond $W 1-W 2=0.3$ ). Therefore strict color limits of $-0.02 \leqslant W 1-W 2 \leqslant 0.17$ have been enforced. The proposed relation is

$$
\log \left(M_{*} / L_{W 1}\right)_{\mathrm{C} 14}=-0.17-2.54 \times(W 1-W 2),
$$

where $M_{*}$ is recovered from the resulting $M_{*} / L_{W 1}$ by multiplying by the corresponding luminosity, $L_{W 1}\left(L_{\odot}\right)=10^{-0.4\left(M_{W 1}-M_{\odot}\right)}$, where $M$ is an absolute magnitude.

Eskew et al. (2012, hereafter E12) proposed an alternate form of a $M_{*}$ conversion, directly calculated from $W 1$ and $W 2$ band fluxes. This transformation was derived by calibrating 3.6 and $4.5 \mu \mathrm{m}$ fluxes to previously existing stellar maps of the Large Magellanic Cloud (LMC). The stellar mass maps by E12 were derived from the detailed star formation history (SFH) maps produced by Harris and Zaritsky (2009), who adopted a Salpeter IMF. With known SFHs, the uncertainty on resulting $M_{*}$ is greatly reduced and strengthens the calibration.

Additionally, with the conversion being calibrated to spatially resolved stellar maps, it can be appropriately applied to the resolved regions of our spiral targets. Like the C14 relation, it has been calibrated over a wide range of environments, albeit increasing uncertainty in strongly SF (warmer) environments. The transformation is given as

$$
M_{\mathrm{E} 12}^{*}\left(M_{\odot}\right)=10^{5.65} F_{W 1}^{2.85}(\mathrm{Jy}) F_{W 2}^{-1.85}(\mathrm{Jy})\left[\frac{D(\mathrm{Mpc})}{0.05}\right]^{2} .
$$

In order to compare this transformation with that of $\mathrm{C} 14$ (Equation (5)), we have recast Equation (6) using a similar notation as Equation (5) (see Appendix B):

$$
\log \left(M_{*} / L_{W 1}\right)_{\mathrm{E} 12}=-0.08-0.74 \times(W 1-W 2) .
$$

The appropriate color range for their transformation is not explicitly specified, though the limits can easily be calculated from their Figures 3 and 4 . We estimate their limits to be $-0.12 \leqslant W 1-W 2 \leqslant 0.34$. This is much broader than those reported by $\mathrm{C} 14$, as seen in Figure 2; however, the resulting $M_{*} / L_{W 1}$ range remains physical. These limits just breach into the range of colors that may result from AGN and strongly SF environments $(W 1-W 2>0.3)$ that Stern et al. (2012) and C14 caution against. Although E12 did not derive this conversion specifically for the WISE bands, we argue that the 3.6 and $4.5 \mu \mathrm{m}$ Spitzer bands are reasonably consistent with the 3.4 and $4.6 \mu \mathrm{m}$ WISE bands. This calibration may thus be applied to WISE, as the respective bands trace the same behavior. A disadvantage of this calibration is the lowmetallicity of to the LMC; thus the conversion mostly applies to similarly low-metallicity regions.

Meidt et al. (2014, hereafter M14) produced an alternate conversion using spatial maps of 2300 galaxies from the Spitzer Survey of Stellar Structure in Nearby Galaxies $\left(S^{4} G\right)$. These maps preserve the structural information that is necessary in our study of the spatially resolved SFMS. Their derivation involves fitting [3.6]-[4.5] $\mu \mathrm{m}$ colors to previously 


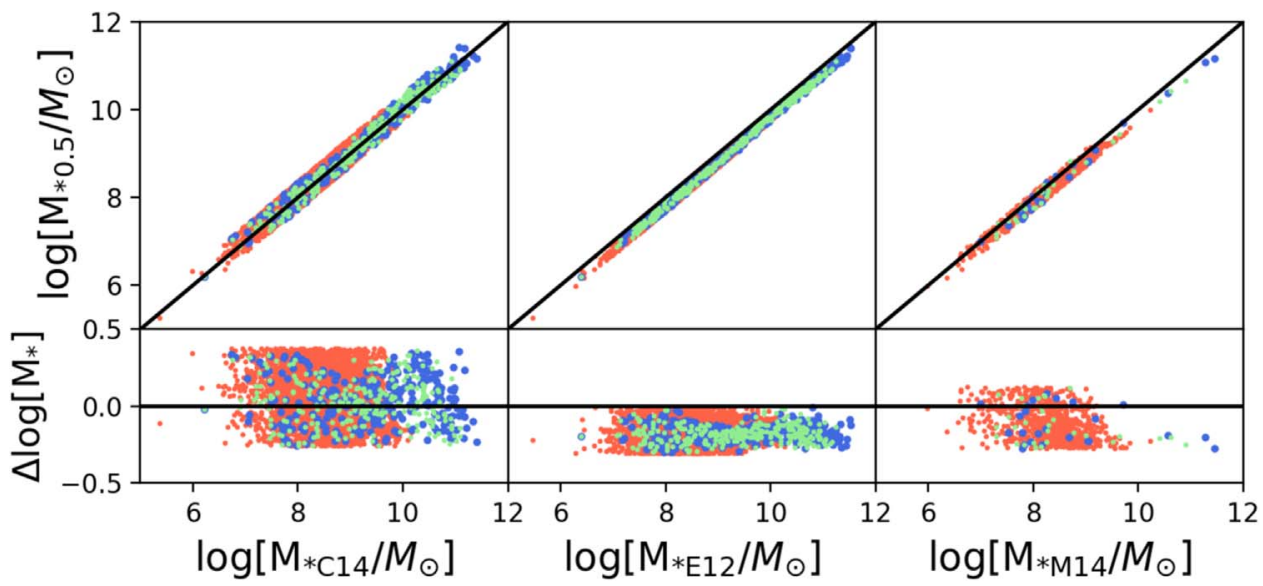

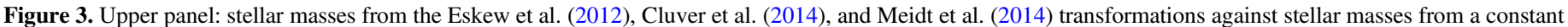

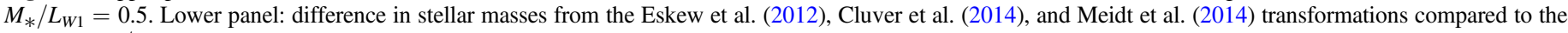

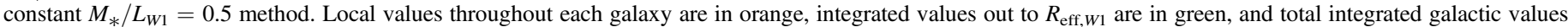
are in blue. The zero difference, or one-to-one, line is overlaid in black.

calculated $M_{*} / L_{3.6}$ 's that assume a Chabrier IMF, with a focus on resolving the age-metallicity degeneracy, which is not accounted for in the E12 study, yielding the relation

$$
\log \left(M_{*} / L_{W 1}\right)_{\mathrm{M} 14}=0.13+3.98 \times(W 1-W 2) .
$$

This calibration, however, requires first removing the contribution of "contaminants" from the detected emission, in order to isolate the old stellar populations. Such contaminants in the [3.6]-[4.5] $\mu \mathrm{m}$ range from hot dust emission, asymptotic giant branch stars to red supergiants. M14 outlines the procedure to eliminate such non-stellar emission by their "Independent Component Analysis" (ICA). To separate purely old stellar populations further, they restrict their study to regions with blue MIR colors, specifically $-0.15<[3.6]-[4.5]<-0.02$. This is consistent with the $W 1-W 2<0$ regime discussed previously for dust-corrected, old stellar populations. This method therefore applies to data sets where dust contributions to MIR emission have been eliminated.

M14 also demonstrate the vulnerability of stellar masses to the adopted IMF. For their preferred Chabrier IMF, they report an average constant $M_{*} / L_{W 1}=0.6$; for a Salpeter IMF, $M_{*} / L_{W 1}$ nearly doubles (1.07). Fortunately, the range of $M_{*} / L_{W 1}$ for each transformation examined here is small and averages about 0.5 . Figure 2 shows CMLRs based on both Chabrier (C14 and M14) and Salpeter (E12) IMFs; the resulting ranges in $M_{*} / L_{W 1}$ show overall consistency.

Numerous studies have advocated for the characterization of a given galaxy stellar population composite by a constant $M_{*} / L$ whose uncertainty encompasses any variations possibly accounted for by CMLRs. The commonly proposed range, $M_{*} / L_{W 1}=0.45-0.6$ (E12, M14, Norris et al. 2014; McGaugh \& Schombert 2015; Kettlety et al. 2018; Ponomareva et al. 2018). For instance, Kettlety et al. (2018) compared stellar masses from SED fits and CMLRs with those derived from a constant $M_{*} / L_{W 1}$, and found matching results. The constant value of $M_{*} / L_{W 1}=0.5$ shown in Figure 2 is clearly a good match to the applicable color range and consistent within the uncertainty associated with stellar mass approximations.

The upper panel of Figure 3 demonstrates the consistency of various $M_{*} / L$ methods, despite the varying MIR color ranges and $M_{*} / L_{W 1}$ trends (Figure 2) among common transformations. Here, stellar masses are compared for regions where the MIR color is appropriate for both $M_{*}$ transformations. Although the scatter about the one-to-one line (in black) is non-negligible, it is quite minimal compared to the uncertainty associated with such transformations. This comparison of stellar mass estimates is similar to that of Drory et al. (2004), and we observe a similar degree of scatter.

Interestingly, the increased discrepancies that arise in SFR estimates when calculating resolved local values versus total galactic values (Figure 1) do not hold for stellar mass estimates. Figure 3, which compares $M_{*}$ values from each method, demonstrates that total integrated $M_{*}$ values (in blue), $M_{*}$ values integrated out to $R_{\text {eff }}$ (in green), and resolved local $M_{*}$ values (in orange) show a tight correlation with the one-to-one line, with no increased scatter in local values. The constant scatter for local and global values is emphasized in the lower panel of Figure 3, suggesting that the increased scatter in the local SFMS (Section 5.2) would be dominated by scatter in SFRs or local processes rather than from stellar masses. The consistency among stellar masses integrated out to $R_{\text {eff }}$ and total integrated values demonstrates that, despite a slight shift between those two, there is no significant growth in $M_{*}$ beyond

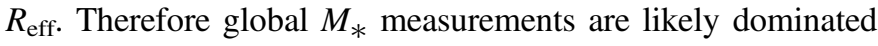
by the inner regions of a galaxy.

In this latter figure, sharp colors limits are imposed in accordance with the $M_{*}$ transformation. Within those regions, the stellar mass estimation methods are clearly consistent. However, beyond these color limits, the physical environments, involving starbursts and AGN activity, are less well constrained, and so are the $M_{*}$ estimates. Such regions are excluded from our study, and hence sharp residual limits are seen in Figure 3.

Since we do not correct for dust emission, dust free and dust polluted regions could potentially be blended. The $W 1-W 2$ colors in each region may serve as an indicator for such emission; if $W 1-W 2<0$, the dust contribution is likely minimal and the M14 transformation should be applied. Conversely, if $W 1-W 2>0$, then dust is likely contributing some of the emission, and either E12 or C14 can be applied.

Such uncertainty justifies the use of a constant $M_{*} / L_{W 1}$ ratio (M14; Norris et al. 2014; Lelli et al. 2016; Kettlety et al. 2018; Ponomareva et al. 2018). Figure 3 highlights that a constant 
Table 1

Star Formation Main Sequence Fit Parameters

\begin{tabular}{|c|c|c|c|c|}
\hline & $\begin{array}{c}\text { SFR } \\
\text { Transformation }\end{array}$ & Slope $a$ & $\begin{array}{c}\text { Zero } \\
\text { Point } b\end{array}$ & $\begin{array}{c}\text { Standard } \\
\text { Deviation } \sigma\end{array}$ \\
\hline Global (G): & Kennicutt (1998b) & 0.79 & -8.32 & 0.31 \\
\hline Local (L): & (Equation (1)) & 0.99 & -10.41 & 0.37 \\
\hline G: & $\begin{array}{l}\text { Cluver et al. } \\
\text { (2017) W3 }\end{array}$ & 1.13 & -11.51 & 0.19 \\
\hline L: & (Equation (2)) & 0.98 & -9.82 & 0.26 \\
\hline G: & $\begin{array}{l}\text { Cluver et al. } \\
\text { (2017) W4 }\end{array}$ & 1.01 & -10.41 & 0.28 \\
\hline L: & (Equation (3)) & 0.94 & -9.64 & 0.35 \\
\hline G: & $\begin{array}{c}\text { Calzetti } \\
\text { et al. (2007) }\end{array}$ & 0.84 & -8.92 & 0.30 \\
\hline L: & (Equation (4)) & 0.99 & -10.26 & 0.30 \\
\hline G: & Davies et al. (2016) & 0.79 & -8.14 & 0.31 \\
\hline L: & $\mathrm{H} \alpha$, old & 0.99 & -10.22 & 0.37 \\
\hline G: & Davies et al. (2016) & 0.45 & -4.35 & 0.21 \\
\hline L: & $\mathrm{H} \alpha$, new & 0.69 & -7.00 & 0.30 \\
\hline G: & Davies et al. (2016) & 0.83 & -8.86 & 0.16 \\
\hline L: & $W 3$, new & 0.79 & -8.45 & 0.20 \\
\hline G: & Davies et al. (2016) & 0.51 & -5.33 & 0.19 \\
\hline L: & $W 4$, new & 0.63 & -6.67 & 0.24 \\
\hline
\end{tabular}

Note. Numerical values for the global and local SFMS fits as plotted in Figure 7 , using a constant $M_{*} / L_{W 1}=0.5$ to calculate the stellar masses, and various transformation methods to determine SFRs. Fits are applied to the global $\log (\mathrm{SFR})=a \log \left(M_{*}\right)+b$ and $\operatorname{local} \log \left(\Sigma_{\mathrm{SFR}}\right)=a \log \left(\Sigma_{M_{*}}\right)+b$ relations. The global and local SFMSs for the Calzetti et al. (2007) SFR transformation (fourth row) are displayed in Figure 6.

$M_{*} / L_{W 1}$ produces fairly consistent stellar masses with all three transformations and appropriate MIR color range. Though stellar masses may be slightly underestimated compared to the E12 and M14 transformations, the resulting values are nonetheless consistent with those estimated in other studies, such as González Delgado et al. (2016) or Ellison et al. (2018; see Figure 6). This study traces considerably more low-mass systems than CALIFA or MaNGA, which are limited to $\log \left(M_{* \text {,tot }} / M_{\odot}\right)>9$; the upper limits in stellar mass are consistent (Walcher et al. 2014; Ellison et al. 2018). Additionally, the constant $M_{*} / L_{W 1}$ is not limited by color range. For this reason, we adopt a constant $M_{*} / L_{W 1}=0.5$ for our $M_{*}$ estimates across the full range of colors.

We have tested for other conversions and obtained statistically consistent SFMS results for the various stellar masses and SFRs (compare Tables 1 and 2).

\section{Spatial Resolution of the SFMS}

The star formation law $\left(\Sigma_{\mathrm{SFR}} \propto \Sigma_{\text {gas }}^{N}\right)$ has received considerable attention (Schmidt 1959; Kennicutt 1998a; Kravtsov 2003; Schaye \& Dalla Vecchia 2008). However, only recently has it become possible to study scales down to $30 \mathrm{pc}$ and address on what physical scales the star formation law holds and possibly even breaks down (Kravtsov 2003; Onodera et al. 2010; Schruba et al. 2010; Kruijssen \& Longmore 2014; Khoperskov \& Vasiliev 2017).

Measurements for the SFMS may no longer be appropriate on scales of individual giant molecular clouds (GMCs),

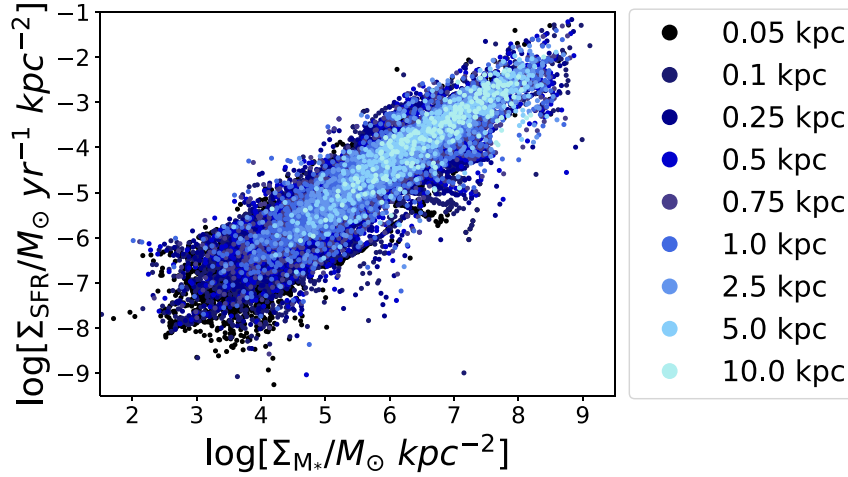

Figure 4. SFMS with $\Sigma_{M_{*}}$ and $\Sigma_{\mathrm{SFR}}$ measurements at various spatial scales. Spatial scales are binned at $50 \mathrm{pc}$ to $10 \mathrm{kpc}$ resolution. The slopes and zeropoints corresponding to local SFMS relations measured on different scales hardly change.

particularly below $100 \mathrm{pc}$. On such small scales, young clusters often drift from their parent GMC, thus disconnecting measurements of $\Sigma_{\text {SFR }}$ from their related $\Sigma_{\text {gas }}$, and ultimately yielding incorrect $M_{*}$ and SFR. Furthermore, the study of individual molecular clouds lacks the sampling completeness of young stellar populations that is often assumed in SFR (and $M_{*}$ ) transformations. SF activity can also vary from cloud to cloud, and since most SFR transformations are generalized to be applied across all environments, sampling of statistically robust and extended SF activity is therefore required.

Kruijssen and Longmore (2014) suggest that the smallest region size that will contain a complete sampling of $\mathrm{SF}$ environments is $490 \mathrm{pc}$. Over such a scale, radiation from SF will propagate over the appropriate timescale for stellar mass growth. They also derive the minimum scale that completely samples IMFs used in most transformation methods to be 340 pc. The maximum spatial scale that young stellar clusters will drift from their parent GMC also appears to be $140 \mathrm{pc}$. The maximum of these spatial scales must therefore be used as the minimum scale over which to apply $M_{*}$ and SFR transformation, in order to produce physically meaningful measurements. Therefore we opt for a minimum spatial resolution of $500 \mathrm{pc}$ in order to produce reliable measurements and probe local variations of $M_{*}$ and SFR within galaxies.

Finally, Boquien et al. (2015) examined SFR transformations on spatial scales from 33 to $2084 \mathrm{pc}$ and posited that monochromatic SFR transformations break down before hybrid transformations with decreasing spatial scales. This further motivates our choice of the $\mathrm{C} 07 \mathrm{H} \alpha+24 \mu \mathrm{m}$ transformation, whose calibration covers a large range of resolutions, over pure $\mathrm{H} \alpha, 23$, or $24 \mu \mathrm{m}$ transformations.

Figure 4 shows our local SFMS binned on scales ranging from 0.05 to $10 \mathrm{kpc}$. The slopes and zero-points of the different SFMS probed at different scales remain essentially the same. The scatter of the SFMS at each resolution scale, shown in Figure 5, is also relatively constant out to $0.5-1 \mathrm{kpc}$, dropping slightly beyond that range. This is broadly consistent with findings by Kruijssen and Longmore (2014), and we also adopt $500 \mathrm{pc}$ as the nominal resolution scale for our study.

\section{The Star Formation Main Sequence}

\subsection{The Global SFMS Relation}

The global SFMS is calculated using total integrated values for each galaxy, which are determined from total enclosed 


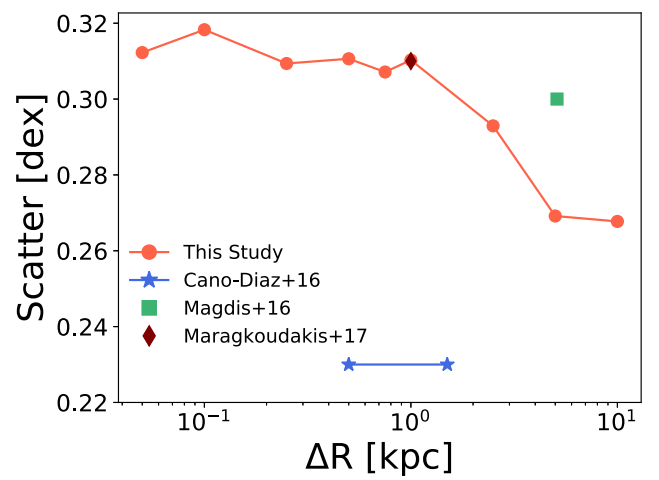

Figure 5. Scatter of the local SFMS measured on various spatial scales (in $\mathrm{kpc}$ ). Our own estimates are shown by orange dots. SFMS scatter estimates by Cano-Díaz et al. (2016), Magdis et al. (2016), and Maragkoudakis et al. (2017) are shown in blue, green, and maroon, respectively.

SINGG and WISE measurements integrated out to the same, matched radius. Multiple transformations were examined, as outlined in Section 3. Figure 6 displays the SFMS constructed with stellar masses using a constant $M_{*} / L_{W 1}=0.5$ and SFRs from the C07 transformations (Equation (4)). Refer back to Sections 3.1 and 3.2 for the motivation for these transformations. The E12 and C14 $M_{*}$ transformations yield essentially the same results (see additional SFMS results in Appendix C).

The top panel of Figure 6 shows the SFMS integrated values are overlayed in blue and green. Local values, plotted in orange in the bottom panel, will be discussed in Section 5.2. The blue points represent the total integrated measurement out to the maximum radius of detection, while green dots are measurements integrated out to $R_{\text {eff. }}$ The blue and green sequences are roughly the same $\left(a_{\text {blue }}=0.84\right.$ vs. $a_{\text {green }}=0.84, b_{\text {blue }}=-8.92$ vs. $b_{\text {green }}=-8.81$, and $\sigma_{\text {blue }}=0.30$ vs. $\sigma_{\text {green }}=0.29$ ), demonstrating that regions beyond $R_{\text {eff }}$ do not considerably affect the global SFMS, and the choice of maximum radius has minimal impact on the global SFMS. In Sections 5.2 and 6 we will compare the SFMS in inner and outer regions. The strong positive linear correlation in the global SFMS already reported by others (e.g., Noeske et al. 2007; Speagle et al. 2014; Salmon et al. 2015) is reproduced here with SINGG and WISE data. Linear fits to the SFMS with the equation $\log (\mathrm{SFR})=a \log \left(M_{*}\right)+b \quad$ using different $\mathrm{SFR}$ and $M_{*}$ transformations are listed in Table 1, and the global fit is overlayed in solid black in Figure 6.

Our measured slopes $(a)$, ranging from about 0.8 to 1.1 , are consistent with those reported by Noeske et al. (2007), Wuyts et al. (2011), Speagle et al. (2014), and Tomczak et al. (2016), accounting for evolutionary factors, excluding fits with the recalibrated $\mathrm{H} \alpha$ and W4 SFR transformations by Davies et al. (2016). The wider range of slopes in Speagle et al. (2014) is largely due to the broad range of environments encompassed, including starbursting to quenched environments. Similarly, the trend of decreasing SFRs with lower redshift (Noeske et al. 2007; Tomczak et al. 2016) results in our magnitude of SFRs, consistent with Wuyts et al. (2011) at $z \approx 0.02-0.2$. However, Table 1 makes clear that our fits, even for the same data set, are not constant. This suggests that the SFMS error budget may be largely spoiled by systematic errors in the adopted transformations, rather than intrinsic environmental factors. The regimes of global SFMS measurements by González Delgado et al. (2016) and Ellison et al. (2018) are outlined in the top panel of Figure 6 in gray, and lie approximately within the same regime as our data, although the

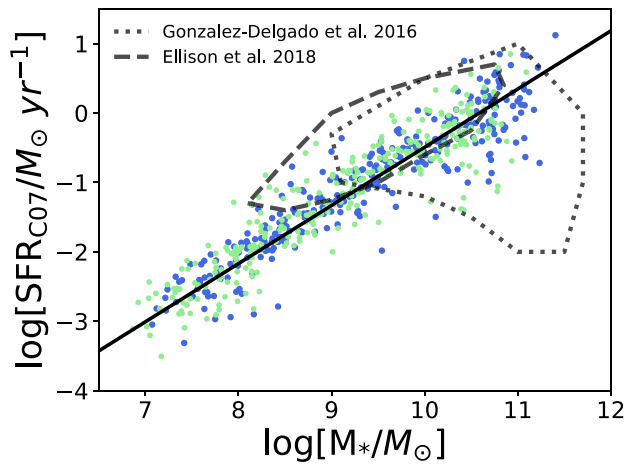

(a)

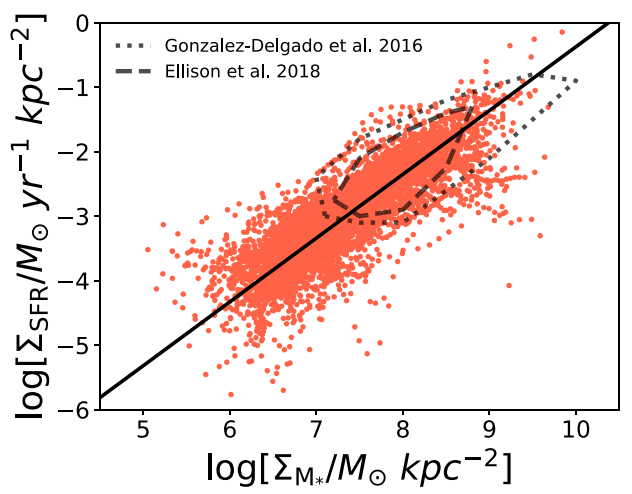

(b)

Figure 6. Global (top) and local (bottom) SFMS. $M_{*}$ values are calculated with a constant $M_{*} / L_{W 1}=0.5$; the SFR are derived from the Calzetti et al. (2007) transformation. Local values for each galaxy are represented as a local density $\left(\Sigma_{M_{*}}, \Sigma_{\mathrm{SFR}}\right)$ and shown in orange, integrated values $\left(M_{*}, \mathrm{SFR}\right)$ out to $R_{\mathrm{eff}}$ are in green, and total integrated galactic values are in blue. Solid lines are the linear fits to global and local values. Fits to the global (blue) and local (orange) SFMS are listed in the fourth row of Table 1. Dashed lines represent the region within the SFMS for measurements found by González Delgado et al. (2016) and Ellison et al. (2018), as indicated in the legend.

CALIFA and MaNGA samples are restricted to $\log \left(M_{*}\right)>$ 9.0 systems (Walcher et al. 2014; Ellison et al. 2018). The CALIFA sample also has a larger contribution of early-type, quiescent galaxies, which accounts for the lower SFR measurements at high $M_{*}$ by González Delgado et al. (2016). While our SFRs are somewhat smaller than those of Ellison et al. (2018), they are broadly consistent with González Delgado et al. (2016), and all agree within the level of uncertainty attached to these transformations (about a factor of two). The higher range of stellar masses show broad consistency with all other considered samples.

The left panel of Figure 7 shows the varying global SFMS fits (slope and intercept) produced using different SFR transformations, as discussed in Section 3.1. Four of them were taken from Davies et al. (2016). With the exception of the solid green and solid blue lines from Davies et al. (2016) for $\mathrm{H} \alpha$ and $W 4$ transformations, the various SFMS fits appear to be highly consistent with each other. However, the calibrations by Davies et al. (2016), originally calibrated for stellar masses above $10^{9} M_{\odot}$, produce unreasonably high SFRs at lower stellar masses. This figure otherwise suggests that the common suite of transformations compares favorably on global, integrated scales. Interestingly, the C17 W3 and W4 transformations have higher SFRs compared to other transformations at high $M_{*}$ values. This could arise from the higher dust content at high SFR and stellar mass, and the $W 3$ and W4 may perform better in this regime. The W3 and W4 

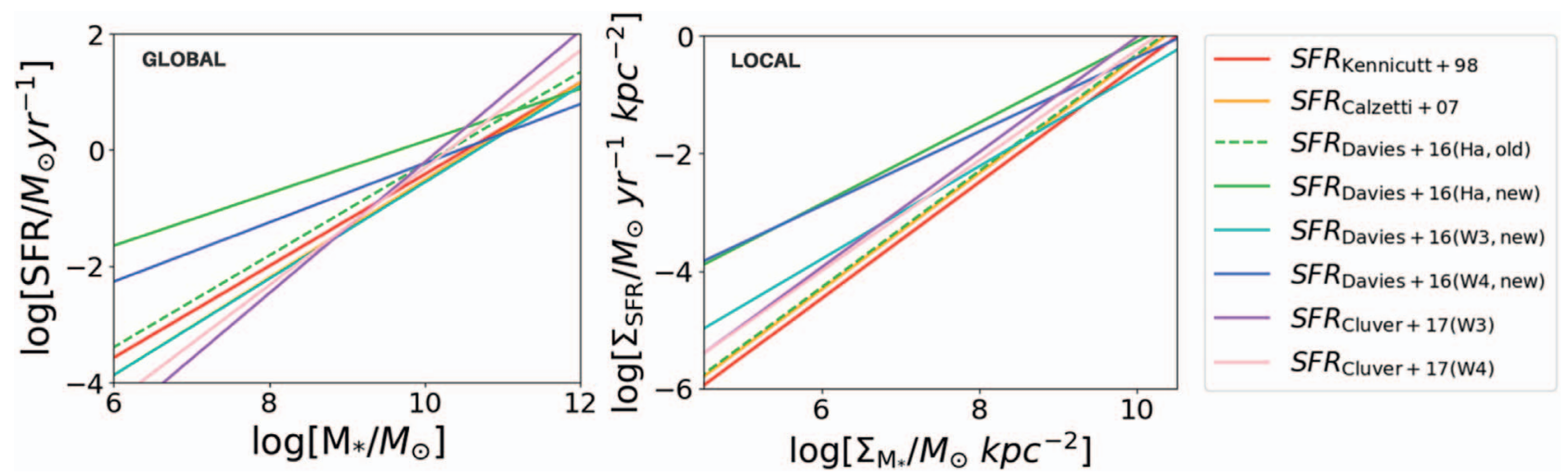

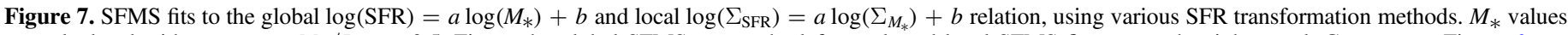
are calculated with a constant $M_{*} / L_{W 1}=0.5$. Fits to the global SFMS are on the left panel, and local SFMS fits are on the right panel. Compare to Figure 6.

transformations could therefore be more robust on global scales, while a hybrid calibration would be more suitable for local measurements.

\subsection{The Local SFMS Relation}

The local SFMS, seen in the bottom panel of Figure 6 in orange, was derived from radially resolved profiles of $\Sigma_{M_{*}}$ and $\Sigma_{\mathrm{SFR}}$ for each galaxy, using transformations by $\mathrm{C} 07$ and C14; the motivation for these is presented in Sections 3 and 5.1. Each measurement represents an annular ring centered around the galactic center, and each ring has a width of $\approx 500 \mathrm{pc}$ by design. The strong positive, linear correlation between SFR and $M_{*}$ typically seen in the the global SFMS is also evident on local $\Sigma_{M_{*}}$ and $\Sigma_{\text {SFR }}$ scales. The local fit in the bottom panel of Figure 6 is shown by black solid line. This further confirms that these two tracers of star formation are strongly coupled not only among galaxies but within themselves; their variations closely track each other. The SFMS regime studied by González Delgado et al. (2016) and Ellison et al. (2018) is again outlined in the bottom panel of Figure 6 in gray, and overlaps with our data. However, unlike the CALIFA and MaNGA samples, significantly lower values of $\Sigma_{M_{*}}$ and $\Sigma_{\mathrm{SFR}}$ measurements are probed in our study thanks to our inclusion of lower stellar mass systems.

Our local SFMS slopes average $a \sim 1$ and are slightly steeper than integrated SFMS slopes (Table 1). They are also consistent, though at the high end, with reported slopes $(a \sim 0.7-1.0)$ from other studies of the spatially resolved SFMS (Pérez et al. 2013; Wuyts et al. 2013; Hemmati et al. 2014; Cano-Díaz et al. 2016; González Delgado et al. 2016; Magdis et al. 2016; Abdurro'uf \& Akiyama 2017; Maragkoudakis et al. 2017). The right panel of Figure 7 shows that SFMS fits from a variety of SFR transformations are highly consistent for local measurements, the exception being the green, cyan, and blue solid lines corresponding to the Davies et al. (2016) relations. This can likely be attributed to the fact that these new calibrations were applied to higher mass systems and were not spatially resolved, whereas the K98 and C07 transformations have been calibrated to such resolved environments. GAMA $\mathrm{H} \alpha$ measurements in Davies et al. (2016) also have a limited aperture size of 2 arcsec that may cause systematic offsets, and the $W 4$ detections were quite poor due to the redshift limits $(z \lesssim 0.13)$. Although environment becomes more stochastic at lower $M_{*}$ regimes, it is interesting that these local SFMS fits and their scatter remain consistent. There is a slight increase in scatter about spatially resolved measurements, and Section 6

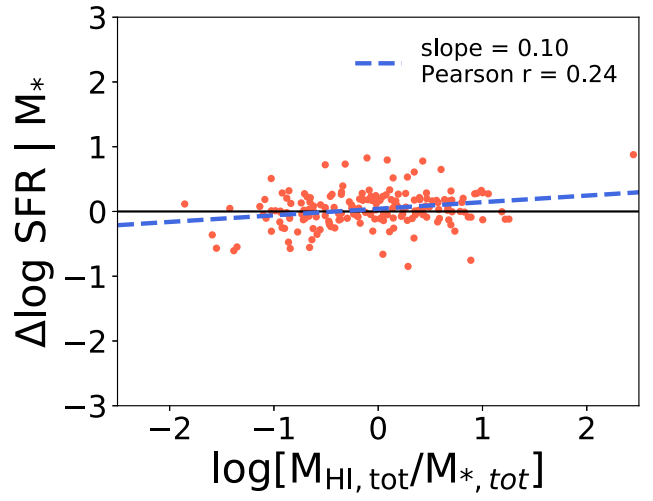

Figure 8. Vertical distance from global SFMS fit $(\Delta \log (\mathrm{SFR}))$ at a fixed $M_{*}$ against the total $\mathrm{H}$ I mass fraction $\left(M_{\mathrm{H} \text { I,tot }} / M_{*, \text { tot }}\right)$. The black line is the linear fit to the SFMS; the blue line is the fit the residuals corresponding to each global parameter. The correlation coefficient is given by Pearson $r$.

investigates the influences by regions within a galaxy versus at a global scale more closely.

\section{SFMS Scatter Analysis}

The main focus of our investigation is to gain a better understanding of the intrinsic scatter of the local, spatially resolved SFMS, since SF shows a strong local scale dependence by the star formation law. The observed scatter about the spatially resolved SFMS is comparable to, though slightly larger than, the scatter about the global SFMS with integrated $M_{*}$ and SFR values, as evidenced from Figure 6 and Table 1. Galaxies in our study are star-forming, by virtue of the SINGG survey selecting galaxies with $\mathrm{HI}$. While the presence of $\mathrm{HI}$ does not directly imply SF, it often correlates with the presence of $\mathrm{H} \alpha$ at a global scale, which is representative of SF, and this is evident in our sample, though we have not imposed that all regions within each galaxy be starforming. While this may contribute to the scatter of the local SFMS, the division between distinct star-forming and quiescent regions that is seen in some global studies of the SFMS (Noeske et al. 2007; Eales et al. 2017; Pandya et al. 2017) does not exist here, as mentioned in Section 5.1. Thus, while SF activity may fluctuate across a galaxy, within a star-forming galaxy, the extremes are not sufficiently large to form two distinct SF sequences.

Cano-Díaz et al. (2016) eliminated regions that do not satisfy the "star-forming conditions" (set by equivalent $\mathrm{H} \alpha$ width measurements, a common criteria for global scale SFMS studies) from their spatially resolved analysis. As such, quiescent regions 


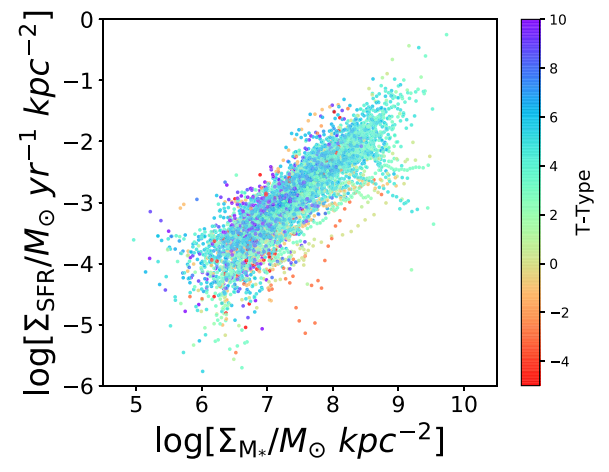

(a)

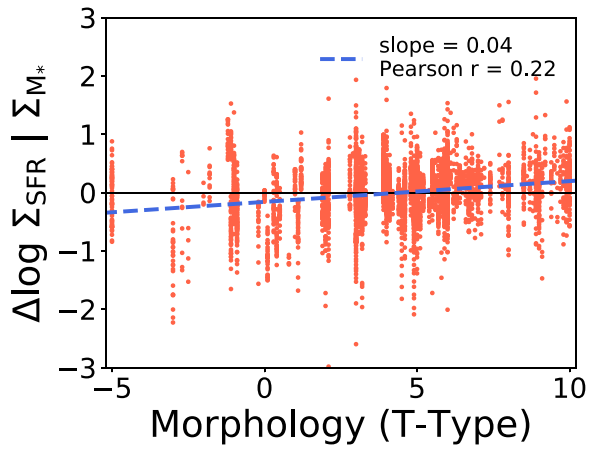

(b)

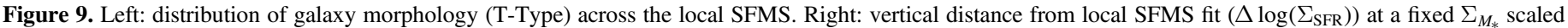

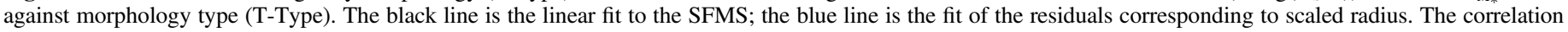
coefficient is given by Pearson $r$.

were not weeded out from our analysis; this may explain the larger (perhaps more physical) scatter that we measure.

Our approach to analyzing the scatter includes the graphical representation of the SFMS as a function of several fundamental parameters and the production of residual figures, emulating the work of Dutton et al. (2007) and Brennan et al. (2017). Key parameters for our scatter analysis include galactic location $\left(R / R_{\mathrm{eff}}\right)$, morphology (T-Type), bulge-to-disk ratio, concentration index, effective surface brightness $\left(\mathrm{SB}_{\mathrm{eff}, W 1}\right)$, inclination, $\Sigma_{\mathrm{SFR}}$, $\Sigma_{M_{*}}$, and so on. Most of these parameters display no strong correlation with the SFMS scatter (see Appendix D for corresponding plots). However, interesting trends with morphological type and radius emerge for individual galaxies.

\subsection{H I Mass Dependence}

We first examine any correlation of the SFMS with the total $\mathrm{H}$ I mass. Our galaxy sample is drawn from the SINGG, which itself targets were HI-rich galaxies selected in HIPASS. Therefore, we wish to assess if this selection biases our sample in any relevant way. Of particular interest is the claim by Saintonge et al. (2016) of a correlation between the ratio of total H I mass to total stellar mass of a galaxy and its position (scatter) within the global SFMS. However, our own examination of the SFMS residuals with H I mass (Figure 8) reveals no such dependence for our sample. This suggests, at least for our sample of star-forming galaxies (with few, if any, quiescent early-type galaxies), that the role of the $\mathrm{H} \mathrm{I}$ mass in regulating star formation is minimal.

\subsection{Morphological Dependence}

It is also necessary to examine any correlation with morphology, as CALIFA SFMS results continue to find strong dependence of the local SFMS scatter on morphology (González Delgado et al. 2016, 2017; López Fernández et al. 2018). According to them, morphology tracks perpendicularly to the SFMS on both global and local scales, suggesting that the host galaxy determines the general SFMS trends (slope and zero point) throughout all regions within the galaxy. While our morphological type coverage is not as extensive, by virtue of the HIPASS sample selection, we do have a broad selection of early-type to late-type to irregular galaxies. Our scatter analysis in fact yields a different trend. Figure 9(a) shows that late-type galaxies (T-Types from 0 to 7) lie throughout the local SFMS, whereas very early-type (T-Type -6 to -3 ) and irregular (T-Type 8 to 10) galaxies appear to dominate in the low $\Sigma_{M_{*}}$ regime. There is no clear orthogonal trend to this relation. This contradictory trend that we find could result from early-type and irregular galaxies still showing a significant amount of observable SF (at least as traced by the presence of HI by HIPASS), but not having enough mass build-up to contribute to the higher mass regions of the SFMS and likely having much smaller systems than typical early-type galaxies. Sample selection is clearly key in establishing the trends observed in our respective analyses of morphological dependence.

\subsection{Radial Dependence}

The radial dependence of the SFMS requires additional scrutiny. Gradient and residual trends are shown in Figures 10 (a) and (b) against the radial parameter, $R / R_{\text {eff }}$. Little correlation is detected in these plots, as is true for most other SFMS residual analyses (again, see Appendix D).

However, patterns emerge when the individual paths of a random selection of galaxies, each distinguished by a unique color, are plotted in Figure 11. These reveal the unique behavior of each galaxy within the SFMS, where a galaxy's central data point is denoted by a star. Galaxies with local mass densities higher than $\log \left(\Sigma_{M_{*}}\right) \sim 7.5$ seem to define two trajectories as their tracks progress from the galactic center to their outskirts. One class (A) initially plateaus in $\Sigma_{\mathrm{SFR}}$ with decreasing $\Sigma_{M_{*}}$. Its radial track then decreases in both $\Sigma_{\mathrm{SFR}}$ and $\Sigma_{M_{*}}$ in lock-step with the SFMS. The alternate class (B) bypasses the initial plateau in $\Sigma_{\mathrm{SFR}}$, plunging from high values of $\Sigma_{\text {SFR }}$ and $\Sigma_{M_{*}}$ in lock-step with the SFMS from the center out. Below $\log \left(\Sigma_{M_{*}}\right) \sim 7.5$, individual galaxy tracks lack structure and show random paths (C). The scale of log $\left(\Sigma_{M_{*}}\right) \sim 7.5$ appears to mark an important transition; whether a galaxy follows the tracks identified above or not, all trends vanish for local SFMS tracks below $\log \left(\Sigma_{M_{*}}\right) \sim 7.5$. This suggests distinctly different mechanisms for high and low-mass density environments. These three galactic behaviors are projected for clarity in the bottom right panel of Figure 11; we label them as the type $\mathrm{A}, \mathrm{B}$, and $\mathrm{C}$ galaxy groups. The schematics given in the bottom right panel of Figure 11 are representative of the broad $\Sigma_{M_{*}}$ and $\Sigma_{\mathrm{SFR}}$ regimes found in our sample; exceptions also exist.

While density measurements eliminate the variation in area among all measurements, so as to better compare different galaxy sizes and internal/radial locations, absolute measurements lack the additional uncertainty introduced from distance errors with density 


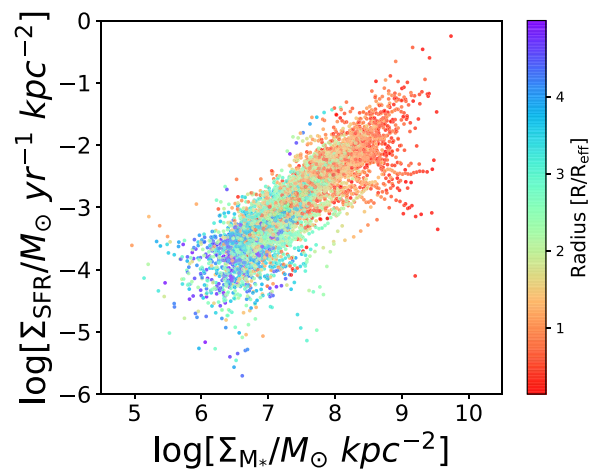

(a)

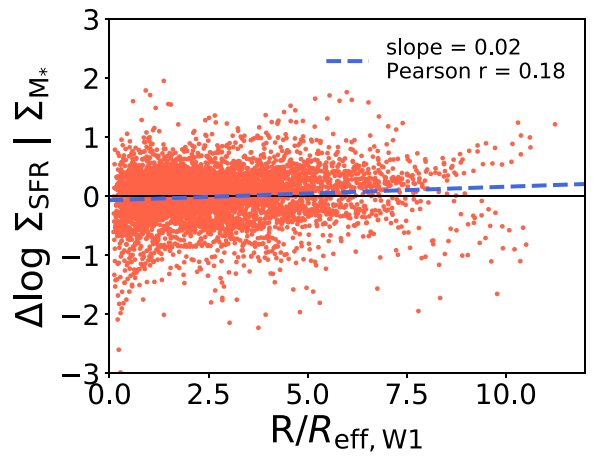

(b)

Figure 10. Left: distribution of radial location $\left(R / R_{\text {eff }}\right)$ within the local SFMS. The gradient displays the range of radial values from $R / R_{\text {eff }}=0$ to $R / R_{\text {eff }}=5$. Right: vertical distance from local SFMS fit $\left(\Delta \log \left(\Sigma_{\mathrm{SFR}}\right)\right)$ at a fixed $\Sigma_{M_{*}}$ scaled against radial value $\left(R / R_{\text {eff }}\right)$. The black line is the linear fit to the SFMS; the blue line is the fit of the residuals corresponding to scaled radius. The correlation coefficient is given by Pearson $r$.
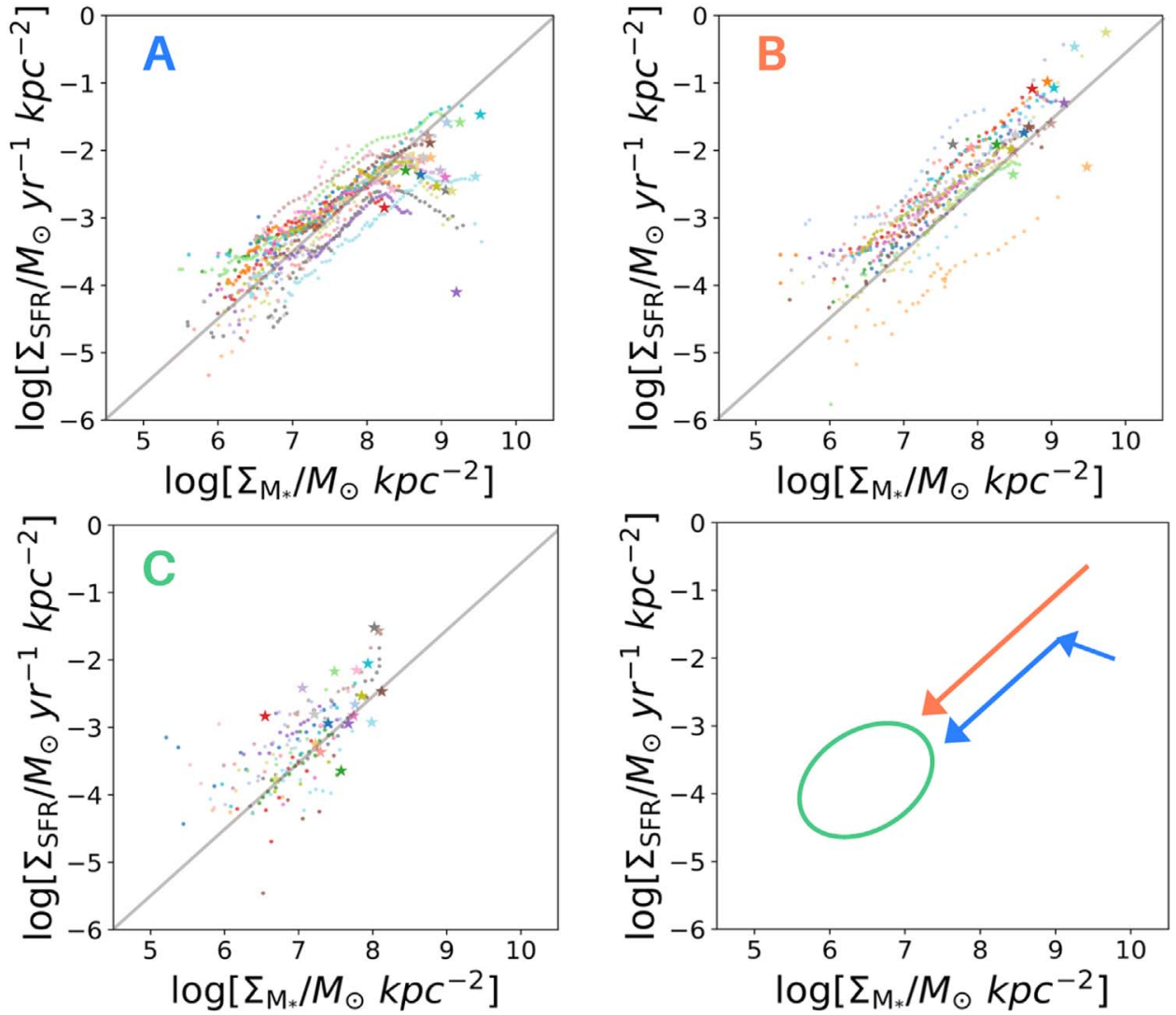

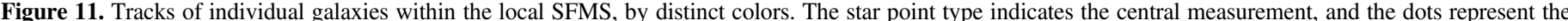

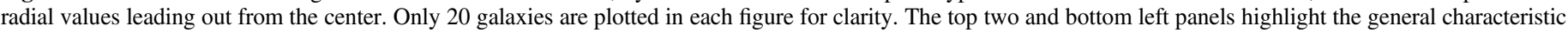

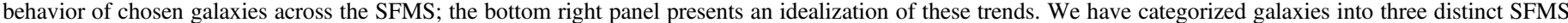
tracks: type A (blue), type B (orange), and type C (green), as depicted in the bottom right panel. Distinct features are discussed in the text.

values. We therefore present in Figure 12 a complementary representation of Figure 11 to further emphasize the distinction between these three types. In this space, the type A population displays an initial sharp rise in the SFR (rather than plateau), whereas type Bs follow the SFMS at all radial points. The stochasticity in type $\mathrm{C}$ measurements is also clear in Figure 12, the $\log \left(\Sigma_{M_{*}}\right) \sim 7.5$ in density space transitions to $\log \left(M_{*}\right) \sim 8.5$ for absolute measurements.

The type A curved tracks provide compelling motivation for inside-out quenching in higher mass systems, as the $\Sigma_{\mathrm{SFR}}$ at the center appears to be significantly reduced compared to the corresponding high stellar mass density. However, we now seek distinguishing features between this and the type B straight, seemingly unquenched, galaxy group that lies in the same local stellar mass density regime. The type B galaxy group generally has higher $\Sigma_{\mathrm{SFR}}$ at a given $\Sigma_{M_{*}}$, as evident in Figure 11.

Another striking feature that emerges from Figure 11 is that the scatter for structures above $\log \left(\Sigma_{M_{*}}\right) \sim 7.5$ appears to be caused by a systematic offset between galaxies. Disregarding 

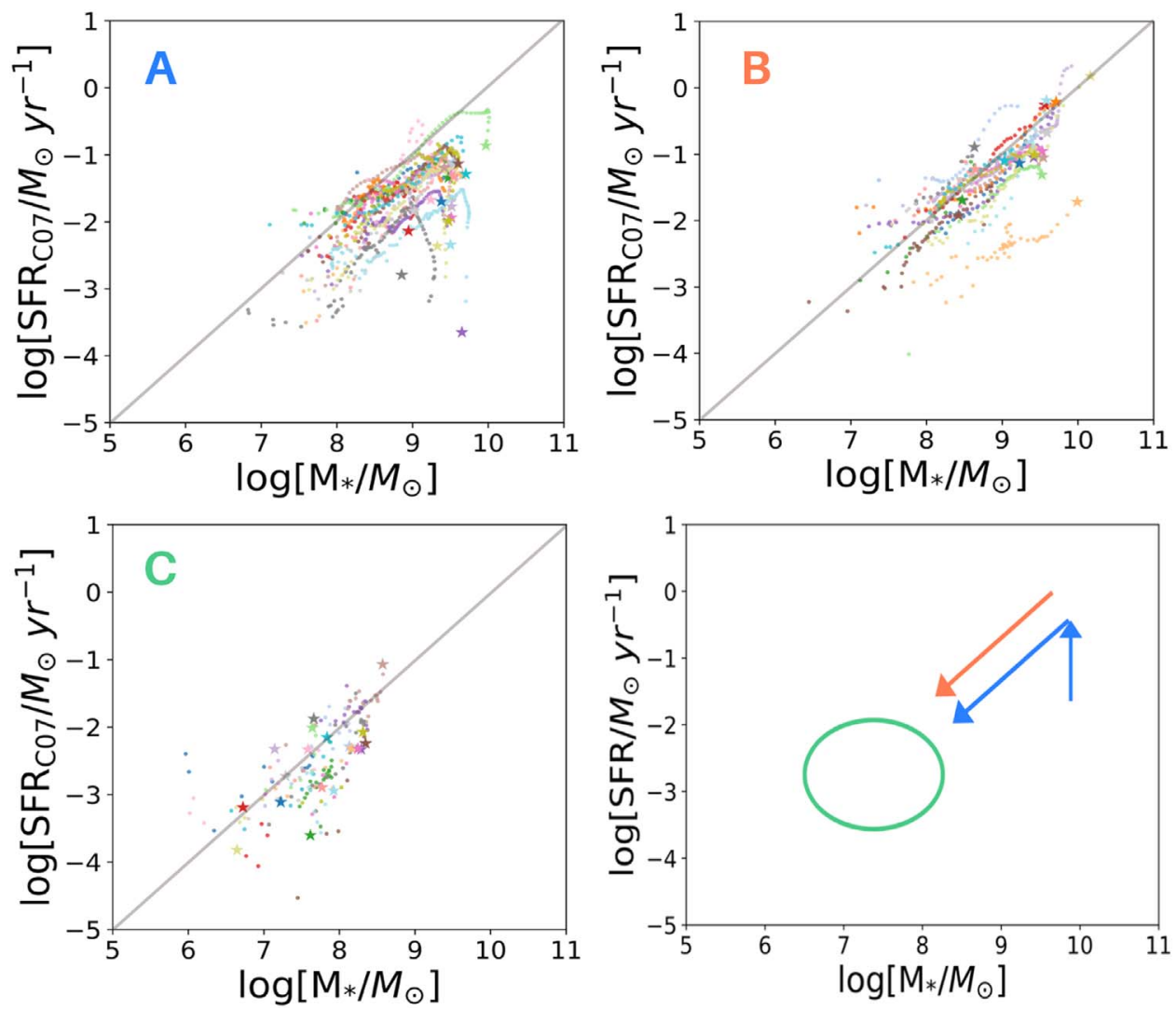

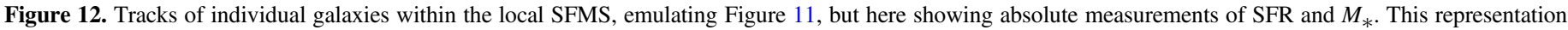

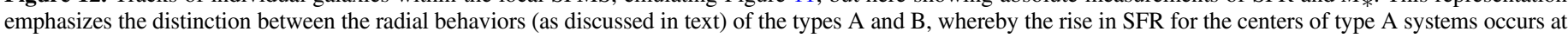
constant stellar mass.

the initial rise in $\Sigma_{\mathrm{SFR}}$ among the type A galaxies, each galaxy appears to lie roughly parallel the SFMS fit, suggesting that the scatter is driven on global scales across galaxies. In fact, applying a linear fit to each individual galaxy track yields consistent slopes $(a)$ with varying intercepts, and the scatter about the SFMS fit associated with an individual galaxy is less than the total scatter of the SFMS, supporting the notion that scatter is dominated by vertical offsets between galaxies, for type $\mathrm{A}$ and $\mathrm{B}$ galaxies.

By contrast, below $\log \left(\Sigma_{M_{*}}\right) \sim 7.5$, SFMS measurements appear to be stochastic in all parts of the galaxies. Interestingly, the overall scatter $(\sim 0.3 \mathrm{dex})$ remains constant at all $\Sigma_{M_{*}}$. It is likely that the random scatter in lower mass density systems is due to stochastic sampling of SF behaviors and environments at such small $\Sigma_{M_{*}}$ scales, and the vertical offsets in type A and B galaxies would be due to varying global accretion rates. The age and accretion histories are difficult to quantify observationally, but stellar mass and SFR-density distributions across a galaxy may potentially inform us of their impact. In this context, a future investigation of star formation and accretion histories with semi-analytic models is certainly warranted.

The global parameters available in this study are still insufficient for the identification of distinguishing features among the three galaxy groups. Out of structural parameters like the concentration index, morphology, bulge-to-total ratio (B/T), and effective surface brightness, only the total stellar and $\mathrm{H}$ I masses $\left(M_{\mathrm{tot}}\right.$ and $\left.M_{\mathrm{H} \mathrm{I}}\right)$ offer any differentiation among the three galaxy groups. Figure 13 shows the A and B type galaxies are generally higher stellar and $\mathrm{H}$ I mass systems, as expected, whereas the stochastic signature of $\mathrm{C}$ types is found in less massive environments. The slight shift upward in total stellar mass $\left(M_{\mathrm{tot}}\right)$ of A type galaxies suggests a more active history of accumulation of stellar mass via higher SFRs in the past. The matching offset in total HI mass is, however, not substantial enough to cleanly delineate the A and B populations. It can be said that As simply have a slightly larger star formation fuel reservoir. No distinction in bulge strength (B/T ratio) was observed between type $\mathrm{A}$ and $\mathrm{B}$ galaxies, though perhaps the activity within the bulge differentiates the two populations, rather than the bulge size itself. Mechanisms such as central mass accretion or SF quenching may also explain the behaviors seen in type A and B galaxies. In order to better understand the different mechanisms distinguishing these galaxy track types, the mean radial profiles of these three groups is examined in Section 7.

\section{Radial Profiles}

Figure 11 shows the type $\mathrm{A}, \mathrm{B}$, and $\mathrm{C}$ tracks of individual galaxies across the local SFMS. We now analyze their mean radial profiles, with a special focus on disentangling the type A and $\mathrm{B}$ populations.

Figure 14 shows radial profiles of $W 1-W 2$ color, $M_{*}, \Sigma_{M_{*}}$, SFR, $\Sigma_{\text {SFR }}$, and sSFR that have been calculated by stacking profiles of galaxies from the same class $(\mathrm{A}, \mathrm{B}$, or $\mathrm{C})$ and scaled to units of the $W 1$ effective radius $\left(R_{\text {eff, } W 1}\right)$. Note that the averages at larger radial values become statistically weaker, as fewer galaxies extend to such radii. We therefore place greater attention to regions within $2.5 R_{\text {eff }}$, as the majority of galaxies in our sample encompass that size. 


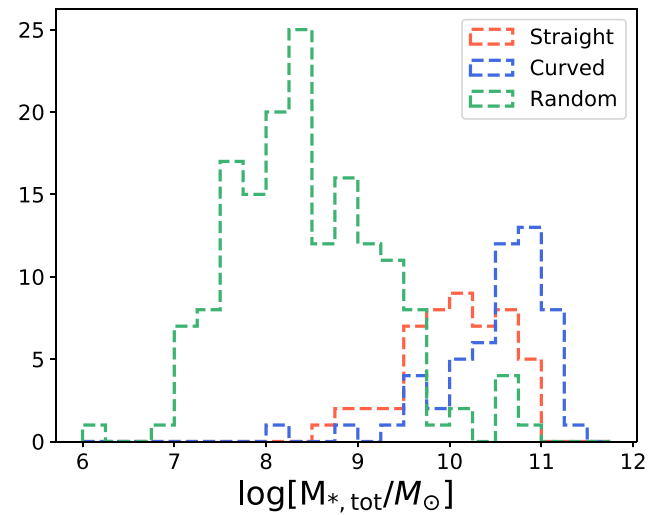

(a)

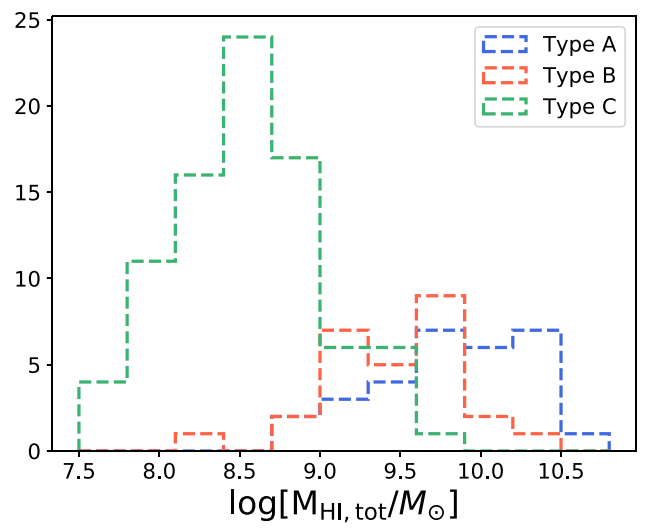

(b)

Figure 13. Distribution of (top) total stellar mass $\left(M_{\mathrm{tot}}\right)$ and (bottom) total $\mathrm{H} \mathrm{I}$ mass $\left(M_{\mathrm{HI}}\right)$ of the three galaxy groups defined in Figure 11.

In Section 6 we posited that either the mass accretion history or SF quenching may separate these galaxy classes. Quenching behavior is generally associated with suppressed SFR relative to the stellar mass growth. This can be defined qualitatively by the sSFR value $\left(\mathrm{SFR} / M_{*}\right)$. High sSFR values may represent systems with active SF (high SFR) compared to the existing stellar mass, whereas a low sSFR may represent systems with a significant amount of $M_{*}$ and/or relatively low SF activity.

The average radial profiles of sSFR values across galaxies may reveal whether galaxies quench from the inside-out or outside-in, if at all. Inside-out quenching (Forbes et al. 2014; González Delgado et al. 2016; Tacchella et al. 2016b; Abdurro'uf \& Akiyama 2018; Belfiore et al. 2018) is dominant in higher stellar mass (Kimm et al. 2009; Pérez et al. 2013) and early-type (Medling et al. 2018) galaxies, and is potentially encouraged in barred galaxies (Abdurro'uf \& Akiyama 2017). Outside-in quenching may be influenced by dense galaxy cluster environments (Schaefer et al. 2017; Medling et al. 2018) or by a lower total stellar mass (Pérez et al. 2013). Typical star-forming sSFR values are higher than $10^{-2} \mathrm{Gyr}^{-1}$, whereas quenched environments have values less than $10^{-2} \mathrm{Gyr}^{-1}$. These limits are not strict, and still debated. For example, Tacchella et al. (2016b) found inside-out quenching, with "quenched" environments measuring $\log (\mathrm{sSFR}) \sim-2$, whereas Medling et al. (2018) identified galaxies with $\log (\mathrm{sSFR}) \sim-3$ to be quenched. We use these thresholds as guidelines and search for "quenched" signatures in our sample, and whether these reflect a suppressed SFR or a higher central build-up of stellar mass.
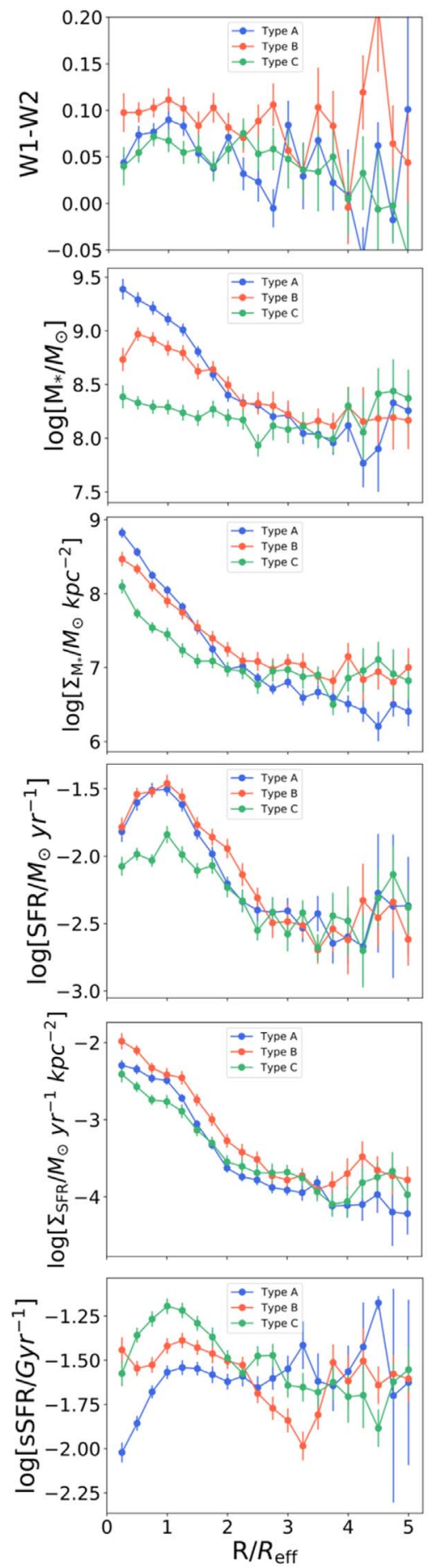

Figure 14. Stacked radial profiles of $W 1-W 2$ color, $M_{*}, \Sigma_{M_{*}}, \mathrm{SFR}, \Sigma_{\mathrm{SFR}}$, and sSFR for galaxies classified in the Type A, B, C galaxy groups (see Figure 11 and Section 6). The $M_{*}$ used to calculate these profiles were derived from a constant $M_{*} / L_{W 1}=0.5$, and SFRs used the Calzetti et al. (2007) transformation. Radial values beyond $R / R_{\text {eff }}=2.5$ become less robust, as fewer galaxies extend that far.

The bottom panel of Figure 14 shows the sSFR profile of the type $\mathrm{A}, \mathrm{B}$, and $\mathrm{C}$ groups. The type A population displays a distinct depression of sSFR within the inner parts of the galaxy, compared to type B and even type C groups. It appears SF activity in class A systems is delayed relative to the amount of stellar mass that has accumulated at the center. Beyond $1-2 R_{\text {eff }}$, the quenching signature dissipates. Similar signatures 
of inside-out quenching are reported in Figure 7 of Tacchella et al. (2016b) and Figure 3 of Belfiore et al. (2018). The latter comparison indicates that the onset of quenching arises first in high stellar mass galaxies. The galaxy is then at a transition point from the star-forming sequence on its way to becoming fully quenched.

Fully quenched galaxies are predominantly early-types (González Delgado et al. 2016; Medling et al. 2018), and since our sample is dominated by spirals and low-mass ellipticals, significantly quenched systems should be less prevalent. However, the type A population seems to feature a transition point for the onset of quenching for late-type galaxies. This signature may very well arise from the presence of a bulge. Indeed, Forbes et al. (2014) suggest that a bulge can quench the inner regions. Type A galaxies will likely reach higher total stellar masses, and thus most likely host a bulge as well (Wang et al. 2017). Similarly, Ellison et al. (2018) propose that central starbursts produce a large central mass build-up, leading to inside-out quenching.

In Figure 14, the SFR profiles of the type A and B populations track each other almost exactly, both with a central dip perhaps indicating a weakly star-forming bulge. This is contrary to expectations from the sSFR profiles, calling for higher SFR measurements from the type B population. This draws attention to the stellar mass profiles. Here, the type A population has significantly higher stellar mass values near the center relative to the type B population. This demonstrates that the "quenched" sSFR signature is in fact the result of a larger accumulation of central mass (and perhaps a longer SFH) in the type A galaxies, while SFR is still ongoing. Another possible factor contributing to the lower $M_{*}$ presence at the center of type Bs may be stellar migration in disks. It is possible that in this galaxy population, mechanisms pushing stars outward in the disk are more dominant, as seen by Frankel et al. (2018).

The $M_{*^{-}}$and SFR-density $\left(\Sigma_{M_{*}}, \Sigma_{\mathrm{SFR}}\right)$ profiles reveal additional clues, along with the pure $M_{*}$ and SFR profiles. The density normalization in the $\Sigma_{M_{*}}$ and $\Sigma_{\mathrm{SFR}}$ measurements shows a decreasing gradient outwards radially in both measurements and no significant suppression of central SF. While the pure $M_{*}$ and SFR profiles highlight nuances to separate these populations, the sSFR profiles can confirm or invalidate the quenching feature. Indeed, the sSFR profile confirms a "transitional" quenching feature in type As, with a large accumulation of mass indicating the now active SF will likely soon slow down. The density profiles also hint at the size variations among the three galaxy populations. Since the density measurements normalize both the $M_{*}$ and SFR values, it suggests that the type A galaxies are generally larger. Therefore the Type $\mathrm{C}$ galaxies are likely smaller in size. This is consistent with Figure 13 and the distribution of total stellar mass associated with each population.

The $W 1-W 2$ color is sensitive to the relative dust content in the region of study, hinting at the remaining fuel for SF. As discussed in Section 3.2, redder colors suggest a larger dust content, whereas blue colors suggest emission by old stellar populations with a minimal contribution from dust. In the $W 1-W 2$ profile, type $\mathrm{B}$ galaxies have, on average, redder $W 1-W 2$ values, thus hinting at a significant dust fraction. This would be consistent with continually active SF regions. In contrast, the bluer $W 1-W 2$ color of type A galaxies suggests they contain an older stellar population. While the SFR profiles of type A and B galaxies may be comparable, the potentially higher dust content implied by $W 1-W 2$ suggests type Bs have a deeper fuel reservoir (dust and gas) to supply ongoing SF for a longer period than type As. This seems consistent with inferences based on the stellar mass profiles, where the type A population has accumulated a considerable amount of stellar mass at the center of the galaxies, and will likely exhaust its fuel before the type B systems.

Conversely, the type $\mathrm{C}$ population has, on average, bluer $W 1-W 2$ colors suggesting lower dust densities, as expected for galaxies of lower total stellar mass (Dalcanton et al. 2004). Lower SFRs for these galaxies scale with lower dust levels in this population, as gas (and dust) would be necessary to fuel the SF activity. sSFRs across type $\mathrm{C}$ galaxies are higher, but this is driven by the drastically lower $M_{*}$ values. The sSFR curve also demonstrates a slight inner quenching signature, though the order of magnitude is still much higher than that of the type A curve, and is likely representative of the sparse stellar population in smaller galaxies, rather than truly quenched environments.

Overall, Figure 14 shows that the onset of inside-out quenching signatures exists in some spiral galaxies, here labeled as type A galaxies. We surmise that the driving factor is not a reduced SFR, but rather a larger accumulation of stellar central mass. This may arise from one or a combination of three scenarios: (1) the galaxy is older, and its SFH is therefore more extended; (2) the galaxy has accreted more mass from external sources in order to fuel continual central SF; or (3) a bar funnels fuel for SF toward the center of the galaxy. The real answer is likely a combination of these three factors, though the first two are difficult to verify observationally. These galaxies may soon transition into the "green valley" range of the SFMS once they have completely exhausted their SF fuel. Finally, type $\mathrm{B}$ and $\mathrm{C}$ galaxies behave as expected for high and lowmass galaxies within the SFMS.

\section{Summary and Conclusions}

We have presented a study of the local and global SFMS and established their comparable behaviors. Using our preferred $M_{*}$ and SFR conversions, we find the fit parameters for the relation $\log (\mathrm{SFR})=a \log \left(M_{*}\right)+b$ to be $a=1.03, b=-10.63$, and $\sigma=0.30$, for the spatially resolved SFMS (see Table 1).

Our study of the SFMS considers radial binning scales down to about $50 \mathrm{pc}$. We have determined the ideal resolution scale to be $500 \mathrm{pc}$ or larger in order to properly encapsulate the physics inherent in the applied transformations.

Our study of the local SFMS uncovered three populations characterized by their radial tracks across the SFMS (Figure 11). These classes, informally referred to as type A, $\mathrm{B}$, and $\mathrm{C}$ galaxies, are characterized as follows:

1. Type A galaxies show a sharp rise in SFR at a relatively constant $M_{*}$ in their inner regions, and then decline with decreasing $M_{*}$ and SFR values along the SFMS slope.

2. Type B galaxies show no sharp rise in SFR at the center, but parallel the SFMS at all radial points also, with finely tuned decreasing $M_{*}$ and SFR values.

3. Type $\mathrm{C}$ galaxies, for which all $\operatorname{local} \log \left(\Sigma_{M_{*}}\right) \sim 7.5$, show no correlation with SFMS within a galaxy, but $M_{*}$ and SFR measurements are more sporadic.

Signatures of inside-out quenching are seen in type A galaxies. This does not appear to arise from suppressed SFR, but rather a larger build-up of stellar mass at the center. We propose that this is likely encouraged by a combination of (1) galaxies being older and therefore having a longer SFH, (2) galaxies having accreted more 


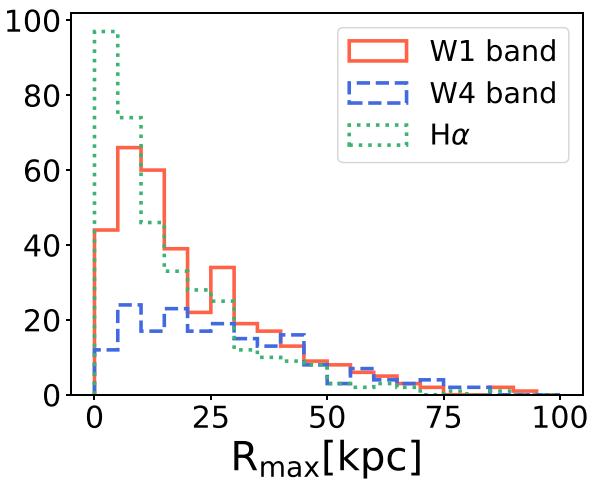

(a)

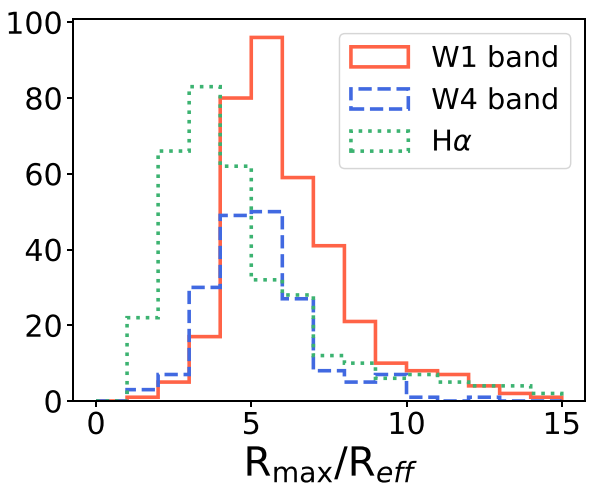

(b)

Figure 15. Radial extent $\left(R_{\max }\right)$ of $\mathrm{H} \alpha$ emission and $W 1$ and $W 4$ band profiles for all galaxies scaled by (a) kiloparsecs (kpc) and (b) effective radius $\left(R_{\text {eff }}\right)$.

mass from external sources to fuel continual SF, or (3) the presence of a bar feeding fuel for SF toward the center. These galaxies may be at a transitionally quenched phase, and as they exhaust their SF fuel, they slide into the "green valley" range of the SFMS (as suggested by Belfiore et al. 2018), perhaps encouraged by central starbursts (as suggested by Ellison et al. 2018).

The scatter in the local SFMS appears to be driven by different mechanisms above and below a critical stellar mass density of log $\left(\Sigma_{M_{*}}\right) \sim 7.5$, yet the same scatter amplitude persists at all stellar mass ranges (in the log-log space of the SFMS). Above local measurements of $\log \left(\Sigma_{M_{*}}\right) \sim 7.5$, type $\mathrm{A}+\mathrm{B}$ galaxies slide along the SFMS slope, but are vertically offset from each other. The scatter in this stellar mass density regime is likely dominated by systematic differences in galaxies and their varying global environments. Below local measurements of $\log \left(\Sigma_{M_{*}}\right) \sim 7.5$, type $\mathrm{C}$ galaxies and even the tails of the type $\mathrm{A}+\mathrm{B}$ galaxies no longer follow the SFMS, but measurements are more sporadic. Scatter is likely dominated by stochastic sampling of SF behavior and environments at such small stellar masses.

The SFMS residual analysis (see also Dutton et al. 2007; Brennan et al. 2017) does not reveal a noticeable correlation with any single structural parameter. In particular, morphology and the $\mathrm{H}$ I gas fraction do not appear to affect the scatter of the global or local SFMS, as suggested by other studies (González Delgado et al. 2016, 2017; Saintonge et al. 2016).

We thank Aaron Dutton and Andrea Macciò for thoughtful discussion enhancing our study. We thank Dennis Zaritsky and Sharon Meidt for insightful conversations about mid-infrared colors and mass-to-light ratios. We thank Richard Tuffs for useful suggestions based on an earlier draft. S.C. acknowledges support from the NSERC of Canada through a Research Discovery Grant.

\section{Appendix A \\ The Radial Extent of Galaxies in Various Bands}

A major advantage of the WISE and SINGG data set is the large extent of the radial profiles. Other surveys such as MaNGA (Albareti et al. 2017) or CALIFA (Sánchez et al. 2016) limit the majority of their galaxies to $1.5 R_{\mathrm{eff}}$ or $4.2 R_{\mathrm{eff}}$, respectively. Figure 15 displays the radial extent of all galaxies in our sample, specifically for $\mathrm{H} \alpha$ emission from SINGG and $W 1$ and $W 4$ band emission from WISE. We rely predominantly on the $\mathrm{H} \alpha$ conversion (Equation (1)), or on the scenario of Equation (4), which uses predominantly $\mathrm{H} \alpha$ and a smaller contribution of $W 4$; regions without $W 4$ emission will represent where there is no re-radiated dust, and SFR is traced by the $\mathrm{H} \alpha$ component.

\section{Appendix B Recasting the $M_{*}$ Transformation}

As discussed in Section 3.2, the $M_{*}$ transformation by E12 (Equation (6)) is given in a very different form than the C14 and M14 transformations. We recast this transformation into the language of the other two forms, as given by Equation (7). Here we outline the procedure to recast the transformation.

First we rearrange the original formula to accommodate distances in pc (no longer Mpc):

$$
\begin{aligned}
M_{\text {Eskew }}^{*} & =10^{5.65} F_{W 1}^{2.85}(\mathrm{Jy}) F_{W 2}^{-1.85}(\mathrm{Jy})\left[\frac{D(\mathrm{Mpc})}{0.05}\right]^{2} \\
& =10^{5.65} F_{W 1}^{2.85}(\mathrm{Jy}) F_{W 2}^{-1.85}(\mathrm{Jy})\left[20 \times \frac{D(\mathrm{pc})}{10^{6}(\mathrm{pc} / \mathrm{Mpc})}\right]^{2} \\
& =10^{-3.75} F_{W 1}^{2.85}(\mathrm{Jy}) F_{W 2}^{-1.85}(\mathrm{Jy}) D^{2}(\mathrm{pc}) .
\end{aligned}
$$

Next, in order to convert the fluxes in Jy to magnitudes, we utilize the "Zero Point Magnitudes" $\left(F_{\nu}(\right.$ iso $\left.)\right)$ reported by Jarrett et al. (2017; the calibration detailed by Jarrett et al. 2011); given a flux density in Jy $\left(S_{\nu}\right)$, the magnitude is determined by $m_{\nu}=-2.5 \times \log _{10}\left[S_{\nu} / F_{\nu}\right.$ (iso) $]$. For the $W 1$ and $W 2$ bands, $F_{W 1}($ iso $)=309.68 \mathrm{Jy}$ and $F_{W 2}($ iso $)=170.66 \mathrm{Jy}$, which results in

$$
\begin{aligned}
M_{\text {Eskew }}^{*}= & 10^{-3.75}\left(309.68 \times 10^{-0.4 m_{W 1}}\right)^{2.85} \\
& \times\left(170.66 \times 10^{-0.4 m_{W 2}}\right)^{-1.85} D^{2}(\mathrm{pc}) \\
= & 10^{-3.75} 309.68^{2.85} 170.66^{-1.85} 10^{-1.14 m_{W 1}} \\
& \times 10^{0.74 m_{W 2}} D^{2}(\mathrm{pc}) \\
\log \left(M_{\text {Eskew }}^{*}\right)= & -0.79-1.14 m_{W 1}+0.74 m_{W 2}+2 \log (D(\mathrm{pc})) .
\end{aligned}
$$

In order to extract the mass-to-light ratio, we must pull $\log \left(L_{\odot, W 1}\right)$ from the right hand side of the above equation. We know that $\log \left(L_{\odot, W 1}\right)=0.4 M_{\odot, W 1}-0.4 M=0.4 M_{\odot, W 1}-$ $0.4 m_{W 1}+2 \log (D(\mathrm{pc}))-2$ (Jarrett et al. 2013; Randriamampandry \& Carignan 2014 report the value $\left.M_{\odot, W 1}=3.24\right)$. 
Table 2

Subsidiary Star Formation Main Sequence Fit Parameters

\begin{tabular}{|c|c|c|c|c|c|}
\hline & $M_{*}$ Transformation & SFR Transformation & Slope $a$ & Zero Point $b$ & Standard Deviation $\sigma$ \\
\hline Global $(\mathrm{G})$ : & Eskew et al. (2012) & Kennicutt (1998b) & 0.79 & -8.43 & 0.31 \\
\hline Local (L): & (Equation (6)) & (Equation (1)) & 1.04 & -10.97 & 0.37 \\
\hline G: & & Cluver et al. (2017) W3 & 1.13 & -11.72 & 0.21 \\
\hline L: & & (Equation (2)) & 1.02 & -10.28 & 0.27 \\
\hline G: & & Cluver et al. (2017) W4 & 1.01 & -10.58 & 0.29 \\
\hline L: & & (Equation (3)) & 0.98 & -10.12 & 0.36 \\
\hline G: & & Calzetti et al. (2007) & 0.83 & -8.96 & 0.30 \\
\hline L: & & (Equation (4)) & 1.02 & -10.74 & 0.31 \\
\hline G: & & Davies et al. (2016) & 0.79 & -8.25 & 0.31 \\
\hline L: & & $\mathrm{H} \alpha$, old & 1.04 & -10.79 & 0.37 \\
\hline G: & & Davies et al. (2016) & 0.45 & -4.43 & 0.21 \\
\hline L: & & $\mathrm{H} \alpha$, new & 0.72 & -7.35 & 0.30 \\
\hline G: & & Davies et al. (2016) & 0.83 & -9.00 & 0.18 \\
\hline L: & & $W 3$, new & 0.82 & -8.79 & 0.21 \\
\hline G: & & Davies et al. (2016) & 0.51 & -5.39 & 0.19 \\
\hline L: & & $W 4$, new & 0.64 & -6.91 & 0.25 \\
\hline G: & Cluver et al. (2014) & Kennicutt (1998b) & 0.78 & -8.21 & 0.35 \\
\hline L: & (Equation (5)) & (Equation(1)) & 1.02 & -10.61 & 0.39 \\
\hline G: & & Cluver et al. (2017) W3 & 1.14 & -11.60 & 0.27 \\
\hline L: & & (Equation (2)) & 0.80 & -8.48 & 0.27 \\
\hline G: & & Cluver et al. (2017) W4 & 1.01 & -10.37 & 0.34 \\
\hline L: & & (Equation (3)) & 0.62 & -6.61 & 0.36 \\
\hline G: & & Calzetti et al. (2007) & 0.83 & -8.74 & 0.34 \\
\hline L: & & (Equation (4)) & 1.10 & -11.04 & 0.33 \\
\hline G: & & Davies et al. (2016) & 0.78 & -8.03 & 0.35 \\
\hline L: & & $\mathrm{H} \alpha$, old & 1.02 & -10.42 & 0.39 \\
\hline G: & & Davies et al. (2016) & 0.44 & -4.26 & 0.23 \\
\hline L: & & $\mathrm{H} \alpha$, new & 0.69 & -7.02 & 0.27 \\
\hline G: & & Davies et al. (2016) & 0.83 & -8.85 & 0.23 \\
\hline L: & & $W 3$, new & 0.80 & -8.48 & 0.23 \\
\hline G: & & Davies et al. (2016) & 0.50 & -5.19 & 0.22 \\
\hline L: & & $W 4$, new & 0.62 & -6.61 & 0.24 \\
\hline
\end{tabular}

Note. Numerical values for the global and local SFMS fits for the alternative stellar mass estimate methods; Eskew et al. (2012) and Cluver et al. (2014). Fits are applied to the global $\log (\mathrm{SFR})=a \log \left(M_{*}\right)+b$ and local $\log \left(\Sigma_{\mathrm{SFR}}\right)=a \log \left(\Sigma_{M_{*}}\right)+b$ relations.

Therefore we can write this equation as

$$
\begin{aligned}
\log \left(M_{\text {Eskew }}^{*}\right)= & -0.79-0.74 m_{W 1}-0.4 m_{W 1} \\
& +0.74 m_{W 2}+2 \log (D(\mathrm{pc}))-2+2 \\
& +0.4 M_{\odot, W 1}-0.4 M_{\odot, W 1} \\
\log \left(M_{\text {Eskew }}^{*}\right)= & -0.79+2-0.4 M_{\odot, W 1}-0.74 m_{W 1} \\
& +0.74 m_{W 2}+\left(0.4 M_{\odot, W 1}-0.4 m_{W 1}\right. \\
& +2 \log (D(\mathrm{pc}))-2) \\
\log \left(M_{\text {Eskew }}^{*}\right)= & -0.074-0.74\left(m_{W 1}-m_{W 2}\right) \\
& +\log \left(L_{\odot}\right) \\
\log \left(M_{\text {Eskew }}^{*}\right)-\log \left(L_{\odot}\right)= & -0.074-0.74(W 1-W 2) \\
\log \left(M_{\text {Eskew }}^{*} / L_{\odot}\right)= & -0.074-0.74(W 1-W 2) .
\end{aligned}
$$

With the stellar mass equation in this form, we can directly compare each method, as seen in Section 3.2.

\section{Appendix C Subsidiary SFMS Fits}

In our main analysis, we have opted for a constant $M_{*} / L_{W 1}=0.5$ to calculate the stellar masses. We now include the SFMS fits produced by the E12 and C14 in Table 2, for completeness. Comparing the various $a, b$, and $\sigma$ values, we find that the choice of $M_{*}$ transformation does not significantly impact the fit of the SFMS.

\section{Appendix D \\ Subsidiary Analysis of Parameters Not Influencing the SFMS Scatter}

We include here, in Figure 16, subsidiary global residual plots that did not indicate any significant correlation of the corresponding parameter to the scatter of the SFMS (see the analysis in Section 6). 


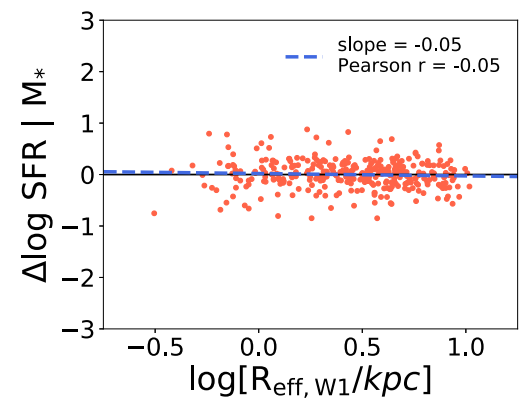

(a)

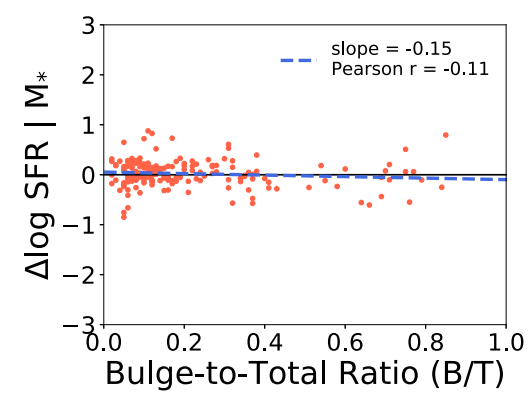

(d)

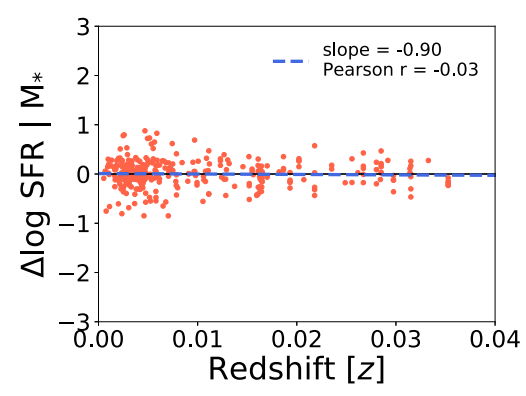

(g)

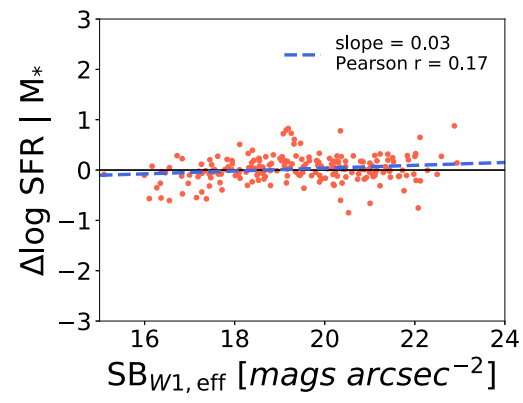

(b)

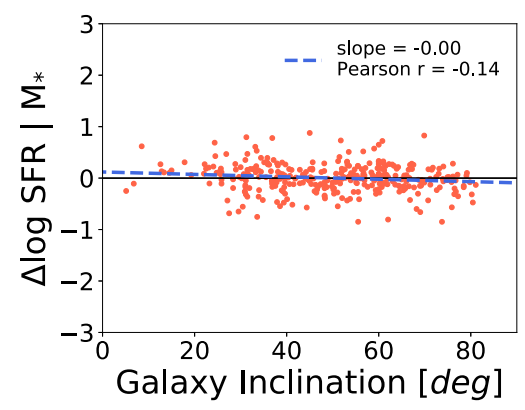

(e)

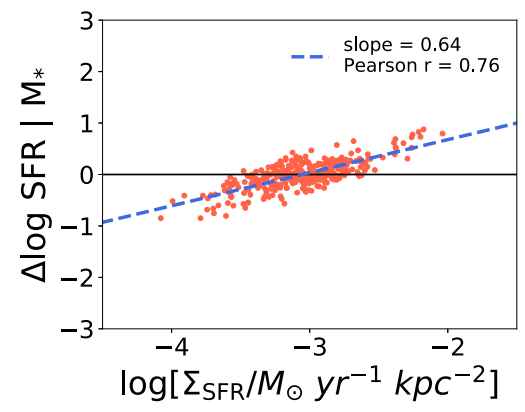

(h)

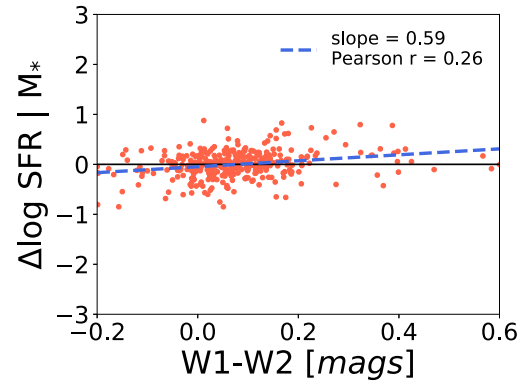

(c)

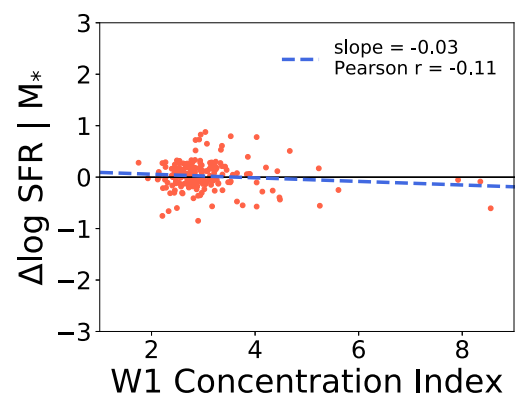

(f)

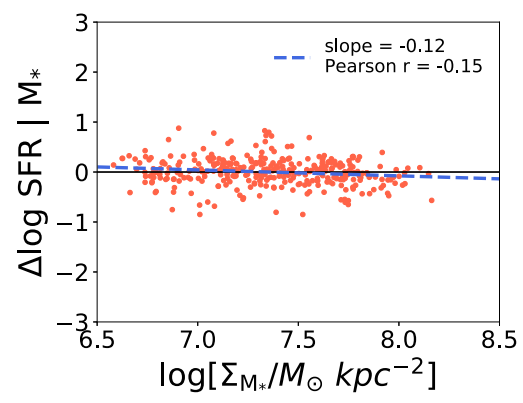

(i)

Figure 16. Vertical distance from global SFMS fit $(\Delta \log (\mathrm{SFR}))$ at a fixed $M_{*}$ against various parameters. The black line is the linear fit to the SFMS; the blue line is the fit the residuals corresponding to each global parameter. The correlation coefficient is given by Pearson $r$.

\section{ORCID iDs}

Stéphane Courteau (ib https://orcid.org/0000-0002-8597-6277

Thomas Jarrett (1) https://orcid.org/0000-0002-4939-734X Michelle Cluver (i) https://orcid.org/0000-0002-9871-6490

\section{References}

Abdurro'uf, \& Akiyama, M. 2017, MNRAS, 469, 2806 Abdurro'uf, \& Akiyama, M. 2018, MNRAS, 479, 5083

Albareti, F. D., Allende Prieto, C., Almeida, A., et al. 2017, ApJS, 233, 25 Belfiore, F., Maiolino, R., Bundy, K., et al. 2018, MNRAS, 477, 3014

Beygu, B., Kreckel, K., van der Hulst, J. M., et al. 2016, MNRAS, 458, 394 Boquien, M., Calzetti, D., Aalto, S., et al. 2015, A\&A, 578, A8

Brennan, R., Pandya, V., Somerville, R. S., et al. 2017, MNRAS, 465, 619

Brown, M. J. I., Jarrett, T. H., \& Cluver, M. E. 2014, PASA, 31, 49B

Calzetti, D. 2013, in Secular Evolution of Galaxies, ed. J. Falcón-Barroso \& J. H. Knapen (Cambridge: Cambridge Univ. Press), 419

Calzetti, D., Kennicutt, R. C., Engelbracht, C. W., et al. 2007, ApJ, 666, $870(\mathrm{C} 07)$

Cano-Díaz, M., Sánchez, S. F., Zibetti, S., et al. 2016, ApJL, 821, L26

Catalán-Torrecilla, C., Gil de Paz, A., Castillo-Morales, A., et al. 2015, A\&A, 584, A87

Cluver, M. E., Jarrett, T. H., Dale, D. A., et al. 2017, ApJ, 850, 68 (C17)

Cluver, M. E., Jarrett, T. H., Hopkins, A. M., et al. 2014, ApJ, 782, 90 (C14)
Coil, A. L., Mendez, A. J., Eisenstein, D. J., \& Moustakas, J. 2017, ApJ, 838,87

Dalcanton, J. J., Yoachim, P., \& Bernstein, R. A. 2004, ApJ, 608, 189

Davies, L. J. M., Driver, S. P., Robotham, A. S. G., et al. 2016, MNRAS, 461, 458

Drory, N., Bender, R., \& Hopp, U. 2004, ApJL, 616, L103

Dutton, A. A., van den Bosch, F. C., \& Dekel, A. 2010, MNRAS, 405, 1690

Dutton, A. A., van den Bosch, F. C., Dekel, A., \& Courteau, S. 2007, ApJ, 654, 27

Eales, S., de Vis, P., Smith, M. W. L., et al. 2017, MNRAS, 465, 3125

Ellison, S. L., Sánchez, S. F., Ibarra-Medel, H., et al. 2018, MNRAS, 474, 2039

Eskew, M., Zaritsky, D., \& Meidt, S. 2012, AJ, 143, 139 (E12)

Forbes, J. C., Krumholz, M. R., Burkert, A., \& Dekel, A. 2014, MNRAS, 438, 1552

Fossati, M., Wilman, D. J., Fontanot, F., et al. 2015, MNRAS, 446, 2582

Frankel, N., Rix, H.-W., Ting, Y.-S., Ness, M. K., \& Hogg, D. W. 2018, arXiv: 1805.09198

Garrison-Kimmel, S., Bullock, J. S., Boylan-Kolchin, M., \& Bardwell, E. 2017, MNRAS, 464, 3108

Genzel, R., Tacconi, L. J., Kurk, J., et al. 2013, ApJ, 773, 68

González Delgado, R. M., Cid Fernandes, R., Pérez, E., et al. 2016, A\&A, 590, A44

González Delgado, R. M., Pérez, E., Cid Fernandes, R., et al. 2017, A\&A, 607, A128

Gu, M., Conroy, C., \& Behroozi, P. 2016, ApJ, 833, 2

Guo, K., Zheng, X. Z., \& Fu, H. 2013, ApJ, 778, 23

Hanish, D. J., Meurer, G. R., Ferguson, H. C., et al. 2006, ApJ, 649, 150 
Harris, J., \& Zaritsky, D. 2009, AJ, 138, 1243

Hemmati, S., Miller, S. H., Mobasher, B., et al. 2014, ApJ, 797, 108

Jarrett, T. H., Cluver, M. E., Magoulas, C., et al. 2017, ApJ, 836, 182

Jarrett, T. H., Cohen, M., Masci, F., et al. 2011, ApJ, 735, 112

Jarrett, T. H., Masci, F., Tsai, C. W., et al. 2012, AJ, 144, 68

Jarrett, T. H., Masci, F., Tsai, C. W., et al. 2013, AJ, 145, 6

Kelson, D. D., Benson, A. J., \& Abramson, L. E. 2016, arXiv:1610.06566

Kennicutt, R. C., Jr. 1983, ApJ, 272, 54

Kennicutt, R. C., Jr. 1998, ApJ, 498, 541

Kennicutt, R. C., Jr. 1998, ARA\&A, 36, 189 (K98)

Kettlety, T., Hesling, J., Phillipps, S., et al. 2018, MNRAS, 473, 776

Khoperskov, S. A., \& Vasiliev, E. O. 2017, MNRAS, 468, 920

Kimm, T., Somerville, R. S., Yi, S. K., et al. 2009, MNRAS, 394, 1131

Kravtsov, A. V. 2003, ApJL, 590, L1

Kruijssen, J. M. D., \& Longmore, S. N. 2014, MNRAS, 439, 3239

Kurczynski, P., Gawiser, E., Acquaviva, V., et al. 2016, ApJL, 820, L1

Lagos, C. d. P., Theuns, T., Schaye, J., et al. 2016, MNRAS, 459, 2632

Lee, J. C., Hwang, H. S., \& Ko, J. 2013, ApJ, 774, 62

Lee, N., Sanders, D. B., Casey, C. M., et al. 2015, ApJ, 801, 80

Lelli, F., McGaugh, S. S., \& Schombert, J. M. 2016, ApJL, 816, L14

Liu, F. S., Jia, M., Yesuf, H. M., et al. 2018, ApJ, 860, 60

López Fernández, R., González Delgado, R. M., Pérez, E., et al. 2018, A\&A, 615, A27

Magdis, G. E., Bureau, M., Stott, J. P., et al. 2016, MNRAS, 456, 4533

Mannucci, F., Cresci, G., Maiolino, R., Marconi, A., \& Gnerucci, A. 2010, MNRAS, 408, 2115

Maragkoudakis, A., Zezas, A., Ashby, M. L. N., \& Willner, S. P. 2017, MNRAS, 466, 1192

Matthee, J., \& Schaye, J. 2018, arXiv: 1805.05956

McGaugh, S. S., \& Schombert, J. M. 2015, ApJ, 802, 18

Medling, A. M., Cortese, L., Croom, S. M., et al. 2018, MNRAS, 475, 5194

Meidt, S. E., Schinnerer, E., van de Ven, G., et al. 2014, ApJ, 788, 144 (M14)

Meurer, G. R., Hanish, D. J., Ferguson, H. C., et al. 2006, ApJS, 165, 307

Mitra, S., Davé, R., Simha, V., \& Finlator, K. 2017, MNRAS, 464, 2766

Noeske, K. G., Weiner, B. J., Faber, S. M., et al. 2007, ApJL, 660, L43

Norris, M. A., Meidt, S., Van de Ven, G., et al. 2014, ApJ, 797, 55

Obreja, A., Brook, C. B., Stinson, G., et al. 2014, MNRAS, 442, 1794

Oemler, A., Jr., Abramson, L. E., Gladders, M. D., et al. 2017, ApJ, 844, 45

Onodera, S., Kuno, N., Tosaki, T., et al. 2010, ApJL, 722, L127

Pandya, V., Brennan, R., Somerville, R. S., et al. 2017, MNRAS, 472, 2054
Pearson, W. J., Wang, L., Hurley, P. D., et al. 2018, A\&A, 615, A146

Peng, Y.-j., Lilly, S. J., Renzini, A., \& Carollo, M. 2012, ApJ, 757, 4

Pérez, E., Cid Fernandes, R., González Delgado, R. M., et al. 2013, ApJL, 764, L1

Ponomareva, A. A., Verheijen, M. A. W., Papastergis, E., Bosma, A., \& Peletier, R. F. 2018, MNRAS, 474, 4366

Querejeta, M., Meidt, S. E., Schinnerer, E., et al. 2015, ApJS, 219, 5

Randriamampandry, T. H., \& Carignan, C. 2014, MNRAS, 439, 2132

Rodríguez-Puebla, A., Primack, J. R., Behroozi, P., \& Faber, S. M. 2016, MNRAS, 455, 2592

Roediger, J. C., \& Courteau, S. 2015, MNRAS, 452, 3209

Rosario, D. J., Mendel, J. T., Ellison, S. L., Lutz, D., \& Trump, J. R. 2016, MNRAS, 457, 2703

Saintonge, A., Catinella, B., Cortese, L., et al. 2016, MNRAS, 462, 1749

Salmon, B., Papovich, C., Finkelstein, S. L., et al. 2015, ApJ, 799, 183

Sánchez, S. F., García-Benito, R., Zibetti, S., et al. 2016, A\&A, 594, A36

Schaefer, A. L., Croom, S. M., Allen, J. T., et al. 2017, MNRAS, 464, 121

Schaye, J., \& Dalla Vecchia, C. 2008, MNRAS, 383, 1210

Schmidt, M. 1959, ApJ, 129, 243

Schruba, A., Leroy, A. K., Walter, F., Sandstrom, K., \& Rosolowsky, E. 2010, ApJ, 722, 1699

Somerville, R. S., Popping, G., \& Trager, S. C. 2015, MNRAS, 453, 4337

Speagle, J. S., Steinhardt, C. L., Capak, P. L., \& Silverman, J. D. 2014, ApJS, 214,15

Stern, D., Assef, R. J., Benford, D. J., et al. 2012, ApJ, 753, 30

Tacchella, S., Dekel, A., Carollo, C. M., et al. 2016a, MNRAS, 457, 2790

Tacchella, S., Dekel, A., Carollo, C. M., et al. 2016b, MNRAS, 458, 242

Tacconi, L. J., Neri, R., Genzel, R., et al. 2013, ApJ, 768, 74

Taylor, E. N., Hopkins, A. M., Baldry, I. K., et al. 2011, MNRAS, 418, 1587

Telford, O. G., Dalcanton, J. J., Skillman, E. D., \& Conroy, C. 2016, ApJ, 827, 35

Tomczak, A. R., Quadri, R. F., Tran, K.-V. H., et al. 2016, ApJ, 817, 118

Walcher, C. J., Wisotzki, L., Bekeraité, S., et al. 2014, A\&A, 569, A1

Wang, W., Faber, S. M., Liu, F. S., et al. 2017, MNRAS, 469, 4063

Whitaker, K. E., Franx, M., Bezanson, R., et al. 2015, ApJL, 811, L12

Whitaker, K. E., Franx, M., Leja, J., et al. 2014, ApJ, 795, 104

Williams, R. J., Quadri, R. F., Franx, M., et al. 2010, ApJ, 713, 738

Wright, E. L., Eisenhardt, P. R. M., Mainzer, A. K., et al. 2010, AJ, 140, 1868

Wuyts, S., Förster Schreiber, N. M., Nelson, E. J., et al. 2013, ApJ, 779, 135

Wuyts, S., Förster Schreiber, N. M., van der Wel, A., et al. 2011, ApJ, 742, 96

Zhang, H.-X., Puzia, T. H., \& Weisz, D. R. 2017, ApJS, 233, 13 